\title{
Transcriptome sequencing of Eucalyptus camaldulensis seedlings subjected to water stress reveals functional single nucleotide polymorphisms and genes under selection
}

\author{
Bala R Thumma ${ }^{1 *}$, Navin Sharma ${ }^{2}$ and Simon G Southerton ${ }^{1}$
}

\begin{abstract}
Background: Water stress limits plant survival and production in many parts of the world. Identification of genes and alleles responding to water stress conditions is important in breeding plants better adapted to drought. Currently there are no studies examining the transcriptome wide gene and allelic expression patterns under water stress conditions. We used RNA sequencing (RNA-seq) to identify the candidate genes and alleles and to explore the evolutionary signatures of selection.

Results: We studied the effect of water stress on gene expression in Eucalyptus camaldulensis seedlings derived from three natural populations. We used reference-guided transcriptome mapping to study gene expression. Several genes showed differential expression between control and stress conditions. Gene ontology (GO) enrichment tests revealed up-regulation of 140 stress-related gene categories and down-regulation of 35 metabolic and cell wall organisation gene categories. More than 190,000 single nucleotide polymorphisms (SNPs) were detected and 2737 of these showed differential allelic expression. Allelic expression of 52\% of these variants was correlated with differential gene expression. Signatures of selection patterns were studied by estimating the proportion of nonsynonymous to synonymous substitution rates (Ka/Ks). The average Ka/Ks ratio among the 13,719 genes was 0.39 indicating that most of the genes are under purifying selection. Among the positively selected genes (Ka/Ks > 1.5) apoptosis and cell death categories were enriched. Of the 287 positively selected genes, ninety genes showed differential expression and 27 SNPs from 17 positively selected genes showed differential allelic expression between treatments.
\end{abstract}

Conclusions: Correlation of allelic expression of several SNPs with total gene expression indicates that these variants may be the cis-acting variants or in linkage disequilibrium with such variants. Enrichment of apoptosis and cell death gene categories among the positively selected genes reveals the past selection pressures experienced by the populations used in this study.

\section{Background}

Abiotic or environmental stresses such as drought, heat, salinity and cold are major impediments to plant survival and productivity in many parts of the world. Plants respond to abiotic stress conditions through diverse biochemical and physiological processes such as accumulation of osmolytes and proteins, reduction in stomatal

\footnotetext{
* Correspondence: reddy.thumma@csiro.au

'CSIRO Plant Industry, Clunies Ross Street, Acton, ACT 2601, Australia

Full list of author information is available at the end of the article
}

conductance, increase in photorespiration and general reduction in growth rate. Osmotic adjustment is one of the common mechanisms of plant response to abiotic stress signals. Water availability is the limiting factor common to all abiotic stresses. As the water potential of the soil water decreases, plants accumulate solutes to reduce the osmotic potential and to maintain water uptake [1]. Several inorganic solutes such as $\mathrm{K}+, \mathrm{Na}+, \mathrm{Cl}$ - and organic solutes such as total soluble sugars, proline, glycine betaine and mannitol are involved in osmotic adjustment. Stress conditions also lead to accumulation of harmful reactive

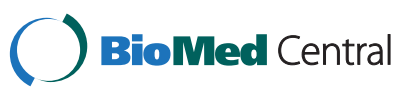


oxygen species (ROS) such as hydroxyl radicals, singlet oxygen, hydrogen peroxide $\left(\mathrm{H}_{2} \mathrm{O}_{2}\right)$ and super oxide $\left(\mathrm{O}_{2}^{-}\right)$. Antioxidant enzymes such as superoxide dismutase (SOD), ascorbate peroxidase (ASX) and catalase help protect plants cells from the harmful effects of ROS [2]. Expression of several detoxification enzymes was shown to increase under stress conditions [3].

Several studies of transcriptional responses to abiotic stress using microarrays [4-8] have identified stress inducible genes that often belong to one of two groups, based on the functions of their gene products. Genes belonging to group 1 are mainly involved in water transport (aquaporins), cellular membrane protection and integrity under stress conditions (proline, glycine betaine, mannitol), scavenging of free oxygen radicals (SOD, catalase), and protecting macromolecules (late embryogenesis abundant proteins - LEA, chaperons). The second group consists of genes that encode regulatory proteins (transcription factors, protein kinases, protein phospatases and calmoduluin binding proteins) and proteins involved in signal transduction $[9,10]$. Stress induced transcription factors are classified into two classes, ABA dependent and ABA independent. The ABA dependent transcription factors include $\mathrm{MYC} / \mathrm{MYB}$ and $A B A$ responsive element binding/ABA binding factor $(\mathrm{AREB} / \mathrm{ABF})$ and the $\mathrm{ABA}$ independent transcription factors are dehydration responsive element binding/Crepeat binding factors (DREB/CBF) belonging to the ethylene responsive factor/APETALA2 (ERF/AP2) family $[9,11]$. The other transcription factors responding to abiotic stress conditions are basic-domain leucine-zipper (bZIP)[12], WRKY binding [13] and NACs [14].

While microarray analyses are useful in revealing genes that are responsive to different conditions, identification of allelic variants from genes showing differential expression may enable their application in breeding by marker-assisted selection. Recent developments in sequencing technology are making it possible to combine gene discovery with identification of allelic variation. Transcriptome sequencing or RNA sequencing (RNAseq) is an approach for quantifying transcripts, in which RNA samples are converted to cDNA and sequenced, typically using high throughput methods. The resulting reads are then mapped against a reference genome sequence or assembled de novo to produce genome-scale transcriptome maps consisting of the structure and abundance of each gene [15]. The abundance of each transcript is determined by counting the number of sequences mapped to the corresponding gene thus providing digital estimate of gene expression. The main advantages of RNA-seq over microarray analysis are a). As RNA-seq is based on counting sequences, cross hybridisation problems associated with microarrays are avoided b). RNA-seq has high dynamic range of detection i.e. very low and very high abundance transcripts can be detected with RNA-seq while microarrays lack sensitivity to detect genes expressed at either high or low levels. Using this technique Zenoni et al. [16] detected several genes expressed during berry development in Vitis vinifera. Similarly several protein coding genes related to xylem formation were identified in an Eucalyptus plantation tree using RNA-seq [17]. RNAseq is also useful for identifying and estimating transcript abundances from alternatively spliced variants [18]. By sequencing several individuals from different populations it is also possible to identify single nucleotide polymorphisms (SNPs) from genes showing differential expression.

In addition transcriptome sequencing can also be used to study the evolutionary selection patterns of genes by estimating nonsynonymous to synonymous substitution (Ka/Ks) ratios. Novaes et al. [19] have shown that most of the genes are under purifying selection by sequencing RNA from different tissues bulked from several individual trees in E. grandis. Combining gene discovery with analysis of selection signatures may provide insights into natural selection patterns under drought stress.

Eucalyptus camaldulensis is one of the most widely planted tree species in the world [20], and is grown extensively in plantations for pulp production in the tropics of South and South East Asia [21,22]. Water availability is the most important factor determining the establishment and composition of tree species in the dry tropics [23]. The seedling stage is the critical period for survival and establishment of trees [24]. In this study we analysed the physiological responses of seedlings of three E. camaldulensis populations subjected to water stress. RNA extracted from leaves of these seedlings was used in RNA-seq analysis to study gene expression patterns under well watered and water stressed conditions. The main objectives of this study are to identify genes differentially expressed under control and stress conditions, to identify allelic variants from these genes and to study the evolutionary signatures of selection.

\section{Results}

\section{Effect of water stress on physiological traits}

Effect of water stress on several physiological and growth traits was analysed by comparing well-watered and water-stressed plants. Two-way ANOVA revealed significant differences between control and stress treatments for all the physiological and biomass traits except for root to shoot ratios (Table 1). While the treatment effect was significant, the population effect was not significant for any of the traits. Similarly no significant interaction between the treatment and population was observed for any of the traits. Pair-wise comparisons 
Table 1 Two-way analysis of variance

\begin{tabular}{|c|c|c|c|c|c|c|c|c|}
\hline Source of Variation & WP & OP & TP & SC & Transpiration & TDM & TE & $R / S$ \\
\hline Population & 0.4736 & 0.7144 & 0.4769 & 0.1398 & 0.9749 & 0.4925 & 0.4042 & 0.4597 \\
\hline Treatment & $<0.0001$ & $<0.0001$ & $<0.0001$ & $<0.0001$ & $<0.0001$ & $<0.0001$ & 0.0028 & 0.268 \\
\hline Interaction & 0.6461 & 0.8215 & 0.3869 & 0.9773 & 0.1565 & 0.1855 & 0.1211 & 0.0983 \\
\hline
\end{tabular}

WP-water potential; OP-osmotic potential; TP-turgor pressure; SC-stomatal conductance; TDM-total dry matter; TE-transpiration efficiency; $R / S$-root to shoot ratio. Sampling 2 (52 days after stress) water relations were analysed.

$P$-values of population, treatment - control vs stress and interaction effects from a two-way analysis of variance of traits.

between the populations for traits were also not significant (data not shown).

\section{Water stress significantly affects Leaf water relations and stomatal conductance}

Leaf water relations were measured on samples collected 30 days (sampling 1) and 52 days (sampling 2) after the imposition of stress treatment. Between the two sampling periods, measurements of water relations (pre-dawn water potential, osmotic potential and turgor pressure) were very similar in control seedlings (Figure 1a, b). However, in stressed seedlings highly significant differences were observed for these traits between the two sampling periods. Within a treatment at both sampling periods, no significant differences were observed between the populations for any of the water relation traits measured (Figure 1a, b). The differences between control and stressed seedlings were much more pronounced 52 days after the imposition of the stress treatment (sampling 2). After 30 days pre-dawn water potentials had decreased to $-0.67 \mathrm{MPa}$ in stressed seedlings compared to $-0.47 \mathrm{MPa}$ in controls. By 52 days pre-dawn water potentials had fallen to $-2.89 \mathrm{MPa}$ and negative turgor pressures were observed in stressed seedlings while in controls these traits were similar to those in sampling 1 .

Mean stomatal conductance was higher in control seedlings (average: $399.45 \mathrm{mmol} \mathrm{m}^{-2} \mathrm{~s}^{-1}$ ) than in water stressed seedlings (average: $239.25 \mathrm{mmol} \mathrm{m}^{-2} \mathrm{~s}^{-1}$ ). Reduction in the stomatal conductance of the Katherine population is higher compared to the other two populations (Figure 1a), however, as with water relations, the stomatal conductance of the three populations were not significantly different (Table 1 ).

\section{Water stress significantly reduces biomass production under stress treatment}

Water stress had a significant effect on all traits related to biomass production (Table 1 ). There was a significant decrease in the amount of water transpired and consequently there was a significant reduction in total dry mass (TDM) produced by stressed seedlings (Additional file 1). The amount of transpiration fell from $49.5 \mathrm{~kg}$ (control) to $14.0 \mathrm{~kg}$ under stress treatment and total biomass produced fell from $112.2 \mathrm{~g}$ (control) to $28.7 \mathrm{~g}$ under stress treatment. Similarly transpiration efficiency (TE-biomass produced per unit of water transpired) decreased from $2.24 \mathrm{~g} / \mathrm{kg}$ in control seedlings to $2.00 \mathrm{~g} / \mathrm{kg}$ in stressed seedlings. Root to shoot ratios of the Katherine and Mt. Isa populations increased while in the Petford population they decreased under stress treatment however, these differences were not significant (Table 1).

\section{RNA sequencing and differential gene expression}

In total, 52 million reads were generated from 12 samples. Reads per sample ranged from two to nine million with an average of 4 million reads per sample. Reads from high throughput sequencing were analysed with TopHat package to develop gene models. Referenceguided mapping was used to predict gene models by mapping the reads against the $E$. grandis reference genome sequence without using E. grandis annotations. By using the coordinates from the predicted gene models we identified the E. grandis genes mapping to the predicted gene regions E. Camaldulensis'. While several of the predicted gene models map to E. grandis gene models there were however several predicted gene models $E$. Camaldulensis that did not map to E. grandis gene models. We used E. grandis gene names wherever the predicted models mapped to the E. grandis models. Where there are no E. grandis annotations mapping to the predicted gene models we used the gene names with a "CUFF" prefix. The coordinates of these genes are presented in Additional file 2.

\section{Reference-guided transcriptome mapping}

Reads from all the 12 libraries were mapped against the Eucalyptus reference genome sequence to generate gene annotations using the TopHat and Cufflinks packages. A total of 32,474 transcripts were predicted including a large number of alternatively spliced transcripts. The identity of the transcripts was investigated by BLAST searches against the Arabidopsis protein database. This analysis revealed 15,538 unique genes from the total transcripts. Read counts mapping to the gene annotations generated by reference-guided transcriptome mapping were used for testing differential expression of the genes between control (C1) and stress (S1) treatments using the edgeR package. Before testing for differential expression, diagnostic tests were performed to test the consistency of the data between the populations. A high 


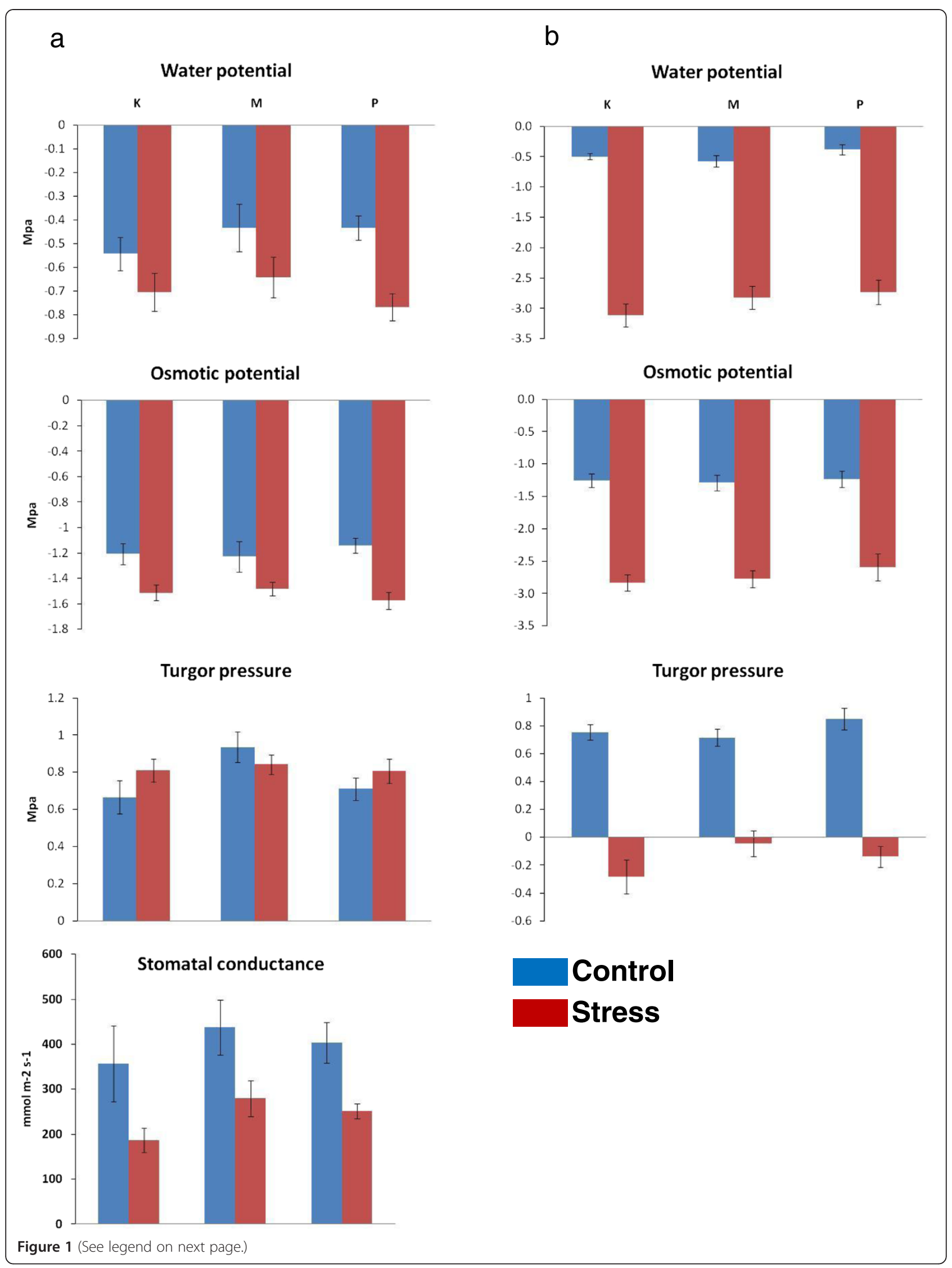


(See figure on previous page.)

Figure 1 Leaf water relations and stomatal conductance in response to water deficit. a). Readings taken 30 days after stress treatment (sampling 1); b). Readings taken 52 days after stress treatment (sampling 2). Stomatal conductance was measured 10 days after stress treatment. Error bars are standard errors of mean (SEM).K-Katherine; M-Mt Isa; P-Petford.

correlation was observed in gene expression between the three populations from a given treatment as measured by the read counts. The Pearson's correlation coefficient between the read counts of the three populations before stress treatment (total six libraries 3 from S0 and three from $\mathrm{C} 0$ ) ranged from 0.94 to 0.99 and the correlation coefficient between the three populations of control plants at the end of the experiment $(\mathrm{C} 1)$ ranged from 0.93 to 0.95 . Similarly in the stress treatment (S1) the correlation coefficients between the populations ranged from 0.94 to 0.97 (Additional file 3). This is further reflected in clustering analysis. Multi-dimensional scaling (MDS) plot of the count data clearly separated the 12 libraries into three groups (Figure 2). The six libraries from the three populations before treatment ( $\mathrm{S} 0$ and $\mathrm{C} 0$ ) were clustered together. Similarly the three populations of control plants $(\mathrm{C} 1)$ at the end of the treatment clustered together while populations from the stress treatment (S1) formed another cluster. As there is a high degree of similarity between the populations from a treatment, reads from each population from a treatment were used as biological replicates in testing for differential expression.

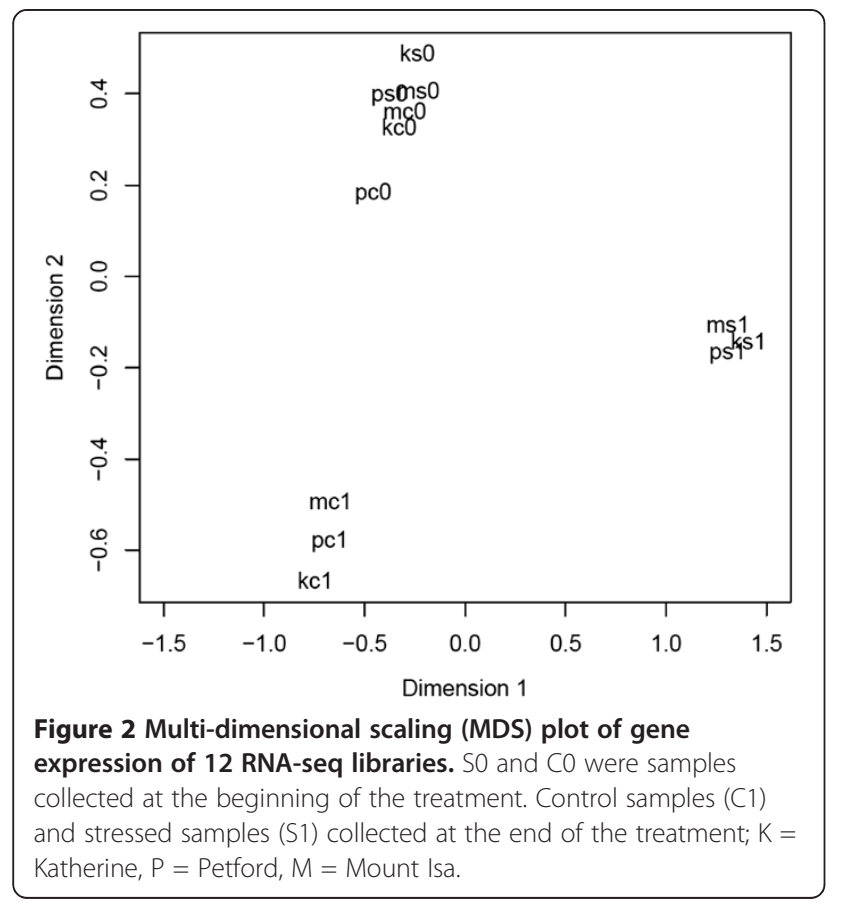

\section{Identification of genes responding to water stress} conditions

To identify genes responding to stress treatment, samples from control (C1) and stress (S1) treatments taken at the end of the stress treatment were analysed for differential gene expression. Analysis of differential gene expression revealed a total of 5270 transcripts (4320 unique genes based on $E$. grandis annotations) that were significantly $(P \leq 0.05$; Bonferroni correction) differentially expressed between the control (C1) and stress (S1) treatments (Additional file 2). Read counts from the three libraries within each treatment are very similar (Table 2). A heatmap of gene expression of the top 200 genes is shown in Figure 3. Variance stabilized data obtained with DESeq pacckage was used to generate the heatmap. The gene expression patterns between the

Table 2 Read counts of significant genes under control and stress treatments

\begin{tabular}{|c|c|c|c|c|c|c|c|c|}
\hline Gene_id & KC1 & MC1 & PC1 & KS1 & MS1 & PS1 & $\log F C$ & FDR \\
\hline Eucgr.K02440 & 145 & 81 & 98 & 0 & 0 & 0 & -35.5 & $2 \mathrm{E}-35$ \\
\hline UFF.8283 & 0 & 0 & 0 & 20 & 4 & 22 & 31.8 & $2 \mathrm{E}-06$ \\
\hline ucgr.L01022 & 0 & 1 & 1 & 82 & 3 & 161 & 5.8 & $8 \mathrm{E}-17$ \\
\hline ucgr.I02271 & 6 & 1 & 43 & 3025 & 1982 & 7510 & 7.1 & $1 \mathrm{E}-62$ \\
\hline icgr.I01041 & 120 & 39 & 138 & 0 & 0 & 0 & -35.3 & $3 E-33$ \\
\hline Etcar & 0 & 2 & 3 & 790 & 199 & 634 & 7.4 & 7E-44 \\
\hline ucgr.J00639 & 2 & 2 & 6 & 248 & 187 & 563 & 5.8 & $5 E-33$ \\
\hline Eucgr.J00493 & 0 & 0 & 0 & 727 & 130 & 181 & 36.4 & $2 E-43$ \\
\hline ucgr.H00163 & 4 & 0 & 1 & 382 & 218 & 461 & 6.9 & $6 \mathrm{E}-39$ \\
\hline ucgr.G01843 & 0 & 0 & 0 & 86 & 47 & 113 & 34.3 & $6 \mathrm{E}-22$ \\
\hline ucgr.A02965 & 10 & 44 & 91 & 0 & 0 & 0 & -34.4 & $3 E-23$ \\
\hline ucgr.F03575 & 89 & 17 & 40 & 0 & 0 & 0 & -34.3 & $1 \mathrm{E}-22$ \\
\hline ucgr.F02915 & 26 & 25 & 90 & 0 & 0 & 0 & -34.3 & $2 \mathrm{E}-22$ \\
\hline ucgr.F02733 & 0 & 1 & 0 & 60 & 96 & 38 & 7.1 & $3 \mathrm{E}-20$ \\
\hline ucgr.F02646 & 467 & 635 & 778 & 0 & 0 & 0 & -38.1 & $2 \mathrm{E}-67$ \\
\hline Eucgr.F01093 & 13 & 16 & 16 & 2534 & 973 & 2670 & 6.2 & $1 \mathrm{E}-49$ \\
\hline JEF 28412 & 0 & 0 & 0 & 843 & 400 & 1068 & 37.5 & $3 \mathrm{E}-56$ \\
\hline Eucgr.F00644 & 2 & 11 & 10 & 4666 & 2578 & 10230 & 8.6 & $2 \mathrm{E}-75$ \\
\hline Eucgr.F00195 & 41 & 44 & 17 & 0 & 0 & 0 & -33.9 & $3 \mathrm{E}-19$ \\
\hline Eucgr.E03257 & 1 & 1 & 3 & 251 & 142 & 436 & 6.5 & $3 E-33$ \\
\hline \multicolumn{9}{|c|}{$\begin{array}{l}M=\text { Mt.Isa; } K=\text { Katherine; } P=\text { Petford; } C 1 \text { - control; S1- stress; logFC - log fold } \\
\text { change; } F D R \text {-false discovery rate. } \\
\text { Read counts of top } 20 \text { most differentially expressed transcripts under control } \\
\text { (C1) and stress conditions (S1). E. grandis gene names are used when the } \\
\text { predicted genes are mapped to E. grandis gene coordinates otherwise the } \\
\text { predicted gene names are used with a pre-fix 'CUFF'. }\end{array}$} \\
\hline
\end{tabular}




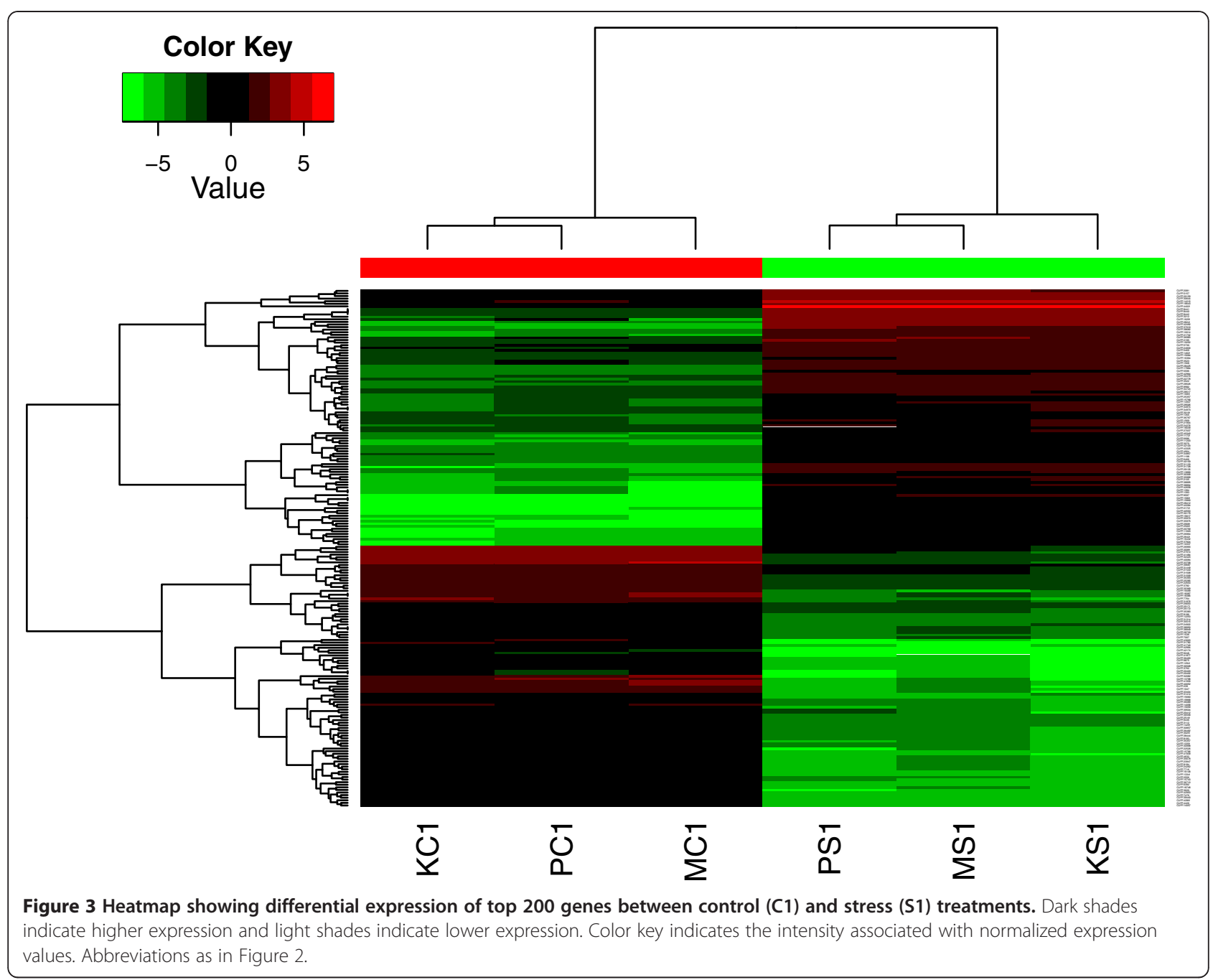

treatments are distinct while within each treatment they are similar. Gene identities of the most differentially expressed transcripts are shown in Table 3. Several heat shock proteins, cell wall genes such as expansins and drought stress related transcription factors ( $H B-12$, $R D 26, E R F 110)$ are among the most strongly differentially expressed genes.

\section{Gene Ontology enrichment analysis}

In order to determine the biological function of differentially expressed genes between control $(\mathrm{C} 1)$ and stress (S1) treatments, gene ontology (GO) based enrichment tests were performed. The top most significantly differentially expressed genes (2642 genes; FDR <1.0e-10) were tested for enrichment using a web-based tool (GOMiner). Arabidopsis homologs of the predicted gene models were obtained by BLAST searches. A total of 175 gene categories were found to be significantly enriched among the genes that were differentially expressed between control (C1) and stress (S1) treatments. Of these,
140 categories were down-regulated, while 35 categories were up-regulated under stress treatment. Within the categories that were up-regulated, most of them were involved in stress response. For example, four of the most significantly enriched gene categories are response to chemical, temperature, heat and abiotic stress stimulus (Table 4). Similarly most of the down-regulated genes belonged to gene categories involved in metabolic processes and cell wall organisation (Table 4).

\section{Identification of growth related genes}

To identify genes relating to growth and development we compared the gene expression between five plants from each population sampled at the beginning of the treatment $(\mathrm{CO})$ and the same five plants sampled at the end of the treatment $(\mathrm{C} 1)$. Gene expression analysis revealed a total of 3582 genes with significant (FDR < 0.01 ) differential expression between $\mathrm{C} 0$ and $\mathrm{C} 1$ samples. To study the expression patterns of these genes under stress conditions we compared the expression of 
Table 3 Gene identities of the most differentially expressed transcripts

\begin{tabular}{|c|c|c|c|c|}
\hline Gene_id & LogFC & FDR & AT_accession & TAIR_gene_annotation ${ }^{1}$ \\
\hline Eucgr.K02440 & -35.5 & $2 \mathrm{E}-35$ & AT3G53190.1 & Pectin lyase-like superfamily protein \\
\hline CUFF.8283 & 31.8 & $2 \mathrm{E}-06$ & & no-hit \\
\hline Eucgr.L01022 & 5.8 & $8 \mathrm{E}-17$ & ATCG00020.1 & photosystem II reaction center protein A \\
\hline Eucgr.I02271 & 7.1 & $1 \mathrm{E}-62$ & AT3G12500.1 & basic chitinase \\
\hline Eucgr.I01041 & -35.3 & $3 \mathrm{E}-33$ & AT3G10450.2 & serine carboxypeptidase-like 7 \\
\hline Eucgr.H04038 & 7.4 & $7 \mathrm{E}-44$ & AT3G62420.1 & basic region/leucine zipper motif 53 \\
\hline Eucgr.J00639 & 5.8 & $5 E-33$ & AT5G21170.2 & 5'-AMP-activated protein kinase \\
\hline Eucgr.J00493 & 36.4 & $2 \mathrm{E}-43$ & AT2G27510.1 & ferredoxin 3 \\
\hline Eucgr.H00163 & 6.9 & $6 \mathrm{E}-39$ & AT2G38940.1 & phosphate transporter $1 ; 4$ \\
\hline Eucgr.G01843 & 34.3 & $6 \mathrm{E}-22$ & AT5G45180.1 & Flavin-binding monooxygenase \\
\hline Eucgr.A02965 & -34.4 & $3 E-23$ & AT5G17050.1 & UDP-glucosyl transferase \\
\hline Eucgr.F03575 & -34.3 & $1 \mathrm{E}-22$ & AT1G32860.1 & Glycosyl hydrolase \\
\hline Eucgr.F02915 & -34.3 & $2 \mathrm{E}-22$ & & no-hit \\
\hline Eucgr.F02733 & 7.1 & $3 \mathrm{E}-20$ & AT1G78780.2 & pathogenesis-related \\
\hline Eucgr.F02646 & -38.1 & $2 \mathrm{E}-67$ & AT5G09360.1 & laccase 14 \\
\hline Eucgr.F01093 & 6.2 & $1 \mathrm{E}-49$ & AT4G27410.3 & RD26 | NAC domain transcriptional regulator \\
\hline CUFF.28412 & 37.5 & $3 \mathrm{E}-56$ & AT4G27450.1 & Aluminium induced protein \\
\hline Eucgr.F00644 & 8.6 & $2 \mathrm{E}-75$ & & no-hit \\
\hline Eucgr.F00195 & -33.9 & $3 \mathrm{E}-19$ & AT5G42830.1 & acyl-transferase \\
\hline Eucgr.E03257 & 6.5 & $3 \mathrm{E}-33$ & AT2G33830.2 & Dormancy/auxin associated \\
\hline Eucgr.D02645 & 6.0 & $8 \mathrm{E}-47$ & AT3G61890.1 & homeobox 12 \\
\hline Eucgr.D02610 & -35.6 & $3 E-36$ & AT1G73880.1 & UDP-glucosyl transferase \\
\hline Eucgr.D02390 & -34.1 & $3 \mathrm{E}-21$ & AT5G49330.1 & myb domain protein \\
\hline Eucgr.A02214 & -34.1 & $1 \mathrm{E}-20$ & AT1G28110.2 & serine carboxypeptidase-like 45 \\
\hline Eucgr.A02209 & -34.4 & $1 \mathrm{E}-23$ & AT1G28110.2 & serine carboxypeptidase-like 45 \\
\hline Eucgr.A02209 & -34.8 & $1 \mathrm{E}-27$ & AT2G33530.1 & serine carboxypeptidase-like 46 \\
\hline Eucgr.D00606 & 7.2 & $1 \mathrm{E}-57$ & AT1G28330.4 & dormancy-associated protein-like 1 \\
\hline Eucgr.A02070 & 5.8 & $5 E-43$ & AT3G04070.1 & NAC domain containing protein 47 \\
\hline Eucgr.C04029 & -33.9 & $2 \mathrm{E}-19$ & AT2G24800.1 & Peroxidase superfamily protein \\
\hline Eucgr.C03801 & -34.9 & $2 \mathrm{E}-28$ & AT1G67290.1 & glyoxal oxidase-related protein \\
\hline Eucgr.C03715 & 5.8 & $3 \mathrm{E}-17$ & AT5G56550.1 & oxidative stress 3 \\
\hline Eucgr.C03715 & 6.7 & $5 E-25$ & & no-hit \\
\hline Eucgr.A01963 & -36.5 & $2 \mathrm{E}-47$ & AT2G42840.1 & protodermal factor 1 \\
\hline Eucgr.C01020 & -34.1 & $1 \mathrm{E}-20$ & AT5G58880.1 & unknown protein \\
\hline Eucgr.A01768 & -35.3 & $6 \mathrm{E}-33$ & AT4G03270.1 & Cyclin D6;1 \\
\hline Eucgr.B03987 & 6.4 & $5 E-57$ & AT1G60470.1 & galactinol synthase 4 \\
\hline Eucgr.B03602 & -34.4 & $9 \mathrm{E}-24$ & & no-hit \\
\hline Eucgr.B01275 & 6.2 & $8 \mathrm{E}-20$ & AT5G18600.1 & Thioredoxin superfamily protein \\
\hline CUFF.12241 & -35.7 & $3 \mathrm{E}-38$ & & no-hit \\
\hline Eucgr.L02741 & -36.1 & $1 \mathrm{E}-42$ & AT5G17040.1 & UDP-Glycosyltransferase superfamily protein \\
\hline Eucgr.K01836 & 10.3 & $6 \mathrm{E}-88$ & AT5G06760.1 & Late Embryogenesis Abundant 4-5 \\
\hline Eucgr.I02392 & 12.7 & $2 \mathrm{E}-85$ & AT2G37670.1 & no-hit \\
\hline Eucgr.C00146 & -10.4 & $2 \mathrm{E}-84$ & AT2G22980.4 & serine carboxypeptidase-like 13 \\
\hline Eucgr.F04160 & -11.7 & $5 E-83$ & AT5G09360.1 & laccase 14 \\
\hline
\end{tabular}


Table 3 Gene identities of the most differentially expressed transcripts (Continued)

\begin{tabular}{|c|c|c|c|c|}
\hline Eucgr.l01495 & 9.5 & 7E-79 & AT3G12500.1 & basic chitinase \\
\hline Eucgr.l02395 & 7.8 & 7E-77 & AT2G21490.1 & dehydrin LEA \\
\hline Eucgr.B02163 & -9.5 & $3 \mathrm{E}-76$ & AT4G28780.1 & GDSL-like Lipase/Acylhydrolase \\
\hline Eucgr.H04427 & -8.7 & 7E-74 & AT3G06350.1 & dehydroquinate dehydratase, putative / shikimate dehydrogenase, putative \\
\hline Eucgr.E00358 & 9.2 & $2 \mathrm{E}-73$ & AT4G17030.1 & expansin-like B1 \\
\hline CUFF.37010 & 8.6 & $4 \mathrm{E}-72$ & AT2G47770.1 & TSPO(outer membrane tryptophan-rich sensory protein) \\
\hline Eucgr.G01342 & -7.6 & $3 \mathrm{E}-71$ & AT4G15480.1 & UDP-Glycosyltransferase superfamily protein \\
\hline Eucgr.L02980 & 7.8 & $2 \mathrm{E}-70$ & AT4G27670.1 & heat shock protein 21 \\
\hline Eucgr.K00423 & 7.9 & $2 \mathrm{E}-70$ & AT4G27670.1 & heat shock protein 21 \\
\hline Eucgr.C01021 & -10.8 & $6 \mathrm{E}-68$ & AT5G17050.1 & UDP-glucosyl transferase 78D2 \\
\hline Eucgr.l01044 & -10.5 & $3 \mathrm{E}-67$ & AT2G22960.1 & alpha/beta-Hydrolases \\
\hline Eucgr.F03917 & -11.7 & $3 \mathrm{E}-66$ & & no-hit \\
\hline Eucgr.l01037 & -7.9 & $3 \mathrm{E}-66$ & AT1G33540.1 & serine carboxypeptidase-like 18 \\
\hline Eucgr.G01113 & -8.3 & $6 \mathrm{E}-66$ & AT1G73290.1 & serine carboxypeptidase-like 5 \\
\hline CUFF.9597 & 11.1 & $2 \mathrm{E}-65$ & AT5G63350.1 & unknown protein \\
\hline Eucgr.H02828 & -7.2 & $6 \mathrm{E}-65$ & AT5G13930.1 & Chalcone and stilbene synthase \\
\hline Eucgr.K02961 & 7.7 & $3 \mathrm{E}-64$ & AT2G40300.1 & ferritin 4 \\
\hline Eucgr.C02812 & -7.2 & $5 E-64$ & AT5G11420.1 & unknown \\
\hline Eucgr.l01954 & -11.7 & $2 \mathrm{E}-63$ & AT1G69530.5 & expansin A1 \\
\hline CUFF.1110 & -7.2 & $5 E-63$ & AT4G16730.1 & terpene synthase 02 \\
\hline Eucgr.A02982 & 8.0 & $1 \mathrm{E}-62$ & & no-hit \\
\hline Eucgr.A02983 & 7.5 & $1 \mathrm{E}-62$ & & no-hit \\
\hline Eucgr.B00944 & -9.3 & $2 \mathrm{E}-62$ & AT1G73620.1 & Pathogenesis-related thaumatin \\
\hline Eucgr.K01196 & -7.6 & $2 \mathrm{E}-62$ & AT1G78570.1 & rhamnose biosynthesis 1 \\
\hline Eucgr.K01402 & 37.9 & $3 \mathrm{E}-62$ & AT5G66780.1 & unknown protein \\
\hline CUFF.15800 & 37.9 & $6 \mathrm{E}-62$ & AT4G16260.1 & Glycosyl hydrolase \\
\hline Eucgr.H02579 & -9.6 & $1 \mathrm{E}-61$ & AT5G09360.1 & laccase 14 \\
\hline Eucgr.A01095 & -7.5 & $2 \mathrm{E}-61$ & AT2G05790.1 & O-Glycosyl hydrolases \\
\hline Eucgr.K00883 & -7.1 & 7E-61 & AT4G03210.2 & xyloglucan endotransglucosylase/hydrolase \\
\hline Eucgr.K02657 & -7.9 & $1 \mathrm{E}-60$ & AT1G75290.1 & NAD(P)-binding Rossmann-fold superfamily protein \\
\hline Eucgr.L01608 & -8.3 & $8 \mathrm{E}-60$ & AT1G73270.1 & serine carboxypeptidase-like 6 \\
\hline CUFF.31133 & 7.6 & $2 \mathrm{E}-59$ & AT1G43730.1 & RNA-directed DNA polymerase (reverse transcriptase) \\
\hline Eucgr.K01402 & 37.7 & $4 \mathrm{E}-59$ & AT5G66780.1 & unknown protein \\
\hline Eucgr.K03589 & -6.8 & $4 \mathrm{E}-59$ & AT5G33370.1 & GDSL-like Lipase/Acylhydrolase \\
\hline Eucgr.H03385 & 8.4 & $6 \mathrm{E}-59$ & AT5G50850.1 & Transketolase family protein \\
\hline Eucgr.H00093 & -7.8 & $4 \mathrm{E}-58$ & AT1G04680.1 & Pectin lyase-like \\
\hline Eucgr.A00203 & -7.4 & $5 \mathrm{E}-58$ & AT3G16910.1 & acyl-activating enzyme 7 \\
\hline Eucgr.F03878 & 7.9 & $6 \mathrm{E}-58$ & AT1G15330.1 & Cystathionine beta-synthase \\
\hline CUFF.38682 & 6.8 & $9 \mathrm{E}-58$ & & no-hit \\
\hline Eucgr.J01348 & 7.7 & $1 \mathrm{E}-57$ & AT5G15250.2 & FTSH protease 6 \\
\hline Eucgr.B03992 & -7.2 & $1 \mathrm{E}-57$ & AT5G49730.1 & ferric reduction oxidase 6 \\
\hline Eucgr.E04218 & -8.2 & $2 \mathrm{E}-56$ & AT5G09360.1 & laccase 14 \\
\hline Eucgr.H00118 & 7.9 & $4 \mathrm{E}-56$ & AT1G04560.1 & AWPM-19-like \\
\hline Eucgr.I00519 & -6.3 & $5 E-56$ & AT4G33510.1 & 3-deoxy-d-arabino-heptulosonate 7-phosphate synthase \\
\hline Eucgr.B02376 & 6.8 & $6 \mathrm{E}-56$ & AT3G46230.1 & heat shock protein 17.4 \\
\hline
\end{tabular}


Table 3 Gene identities of the most differentially expressed transcripts (Continued)

\begin{tabular}{lrlll}
\hline Eucgr.F04203 & 9.4 & $6 \mathrm{E}-56$ & AT5G50080.1 & ethylene response factor 110 \\
Eucgr.G01115 & -7.5 & $2 \mathrm{E}-55$ & AT4G12910.1 & serine carboxypeptidase-like 20 \\
Eucgr.A02790 & 6.3 & $3 \mathrm{E}-55$ & AT5G52300.2 & CAP160 protein \\
Eucgr.C01388 & -6.5 & $5 \mathrm{E}-55$ & AT2G10940.2 & Bifunctional inhibitor/lipid-transfer protein \\
Eucgr.H04428 & -6.3 & $2 \mathrm{E}-54$ & AT3G06350.1 & dehydroquinate dehydratase, putative / shikimate dehydrogenase \\
Eucgr.102267 & 7.0 & $4 \mathrm{E}-54$ & AT3G12500.1 & basic chitinase \\
Eucgr.B03602 & -7.2 & $5 \mathrm{E}-54$ & AT3G13510.1 & Unknown Function \\
Eucgr.C00148 & -9.3 & 5E-53 & AT3G10450.2 & serine carboxypeptidase-like 7 \\
\hline
\end{tabular}

${ }^{1}$ The Arabidosis Information Resource.

Gene models predicted from reference-guided mapping were BLAST searched against Arabidopsis protein data base. Arabidopsis homologs of top significant genes are presented. E. grandis gene names are used when the predicted genes are mapped to $E$. grandis gene coordinates otherwise the predicted gene names are used with a pre-fix 'CUFF'. "CUFF" gene coordinates, read counts and gene identities of all significantly ( $P<0.05$; bonferroni correction) differentially expressed transcripts are presented in the Additional file 2.

significant genes $(F D R<0.01)$ from this analysis with those showing significant differential expression between control $(\mathrm{C} 1)$ and stress (S1) treatments. In total there were 2225 genes common between the two analyses. A significant and negative correlation $(P<0.001 ; r=$ -0.37) was observed in log fold change of the gene expression between the two analyses indicating down regulation of several of the growth genes under stress treatment. Genes showing large fold changes in $\mathrm{C} 1$ versus $\mathrm{S} 1$ and $\mathrm{C} 0$ versus $\mathrm{C} 1$ comparison are shown in Table 5. While several photosynthetic and metabolic process related genes exhibited opposite signs in fold change, basic chitinase, NAC transcription factor and homeo box genes exhibited positive sign between the two comparisons. Gene ontology analysis reflected the down regulation of growth genes under stress conditions. Several metabolic process related gene categories such as 'phenylpropanoid_metabolic_process', 'secondary_metabolic_process' and 'flavonoid_biosynthetic_process' were up-regulated in $\mathrm{C} 0$ versus $\mathrm{C} 1$ comparison (Additional file 4) and the same gene categories were down-regulated in $\mathrm{C} 1$ versus S1 comparison (Table 4). However several stress response gene categories were up-regulated under both the comparisons.

\section{Differential allelic expression}

To study the regulatory variants responding to water stress treatment we measured allelic expression. For this the ten individuals sampled at the beginning of the treatment (S0) and the same ten individual sampled at the end of the stress treatment (S1) were used. Allelic expression of an individual should remain the same even when the total expression of a gene changes. Any change in the allelic expression may indicate the influence of regulatory variants. We observed several SNPs as ten individuals in each population were sequenced. To increase the coverage and confidence of the SNP calls, we combined the reads of the three populations from each treatment (30 trees from each treatment). Using a minimum coverage of 8 reads and a minimum frequency of 0.01, we identified 298,561 SNPs within S0 samples and 483,116 SNPs within the same samples under the stress treatment (S1). There were 196,375 SNPs common to both treatments. Most of the unique SNPs from either treatment generally had low coverage. Allele frequency differences between S0 and S1 treatments were used to identify differential allelic expression (DAE). This analysis revealed 2737 (FDR, P <0.05) SNPs (from 1261 genes) with significant differences in allelic expression between the two treatments (Additional file 5). Among these SNPs 68\% were transition substitutions while $32 \%$ were transversion substitutions. Chitinase, zinc finger, plastocynin and cellulose synthase had large differences in allelic expression between the two treatments (Table 6). Allelic expression of 52\% of SNPs (1427 SNPs) correlated with differential gene expression suggesting that these may be the cis-acting regulatory variants controlling gene expression. Genes with significant differences in allelic expression and total gene expression include Chitinase, heat repeat containing protein, and Dehydrin. Allelic expression of the remaining $48 \%$ of the SNPs (1310 SNPs) did not correlate with total gene expression. Several heat shock protein genes were present among this group (Additional file 5). The number of variants showing differential allelic expression was generally biased towards genes with high coverage and alleles with large expression differences between the treatments.

Using E. grandis gene annotations we classified the SNPs as three-prime, synonymous, nonsynonymous, five-prime and intronic SNPs. Synonymous and nonsynonymous SNPs were annotated using PoPoolation package [25]. While most of the SNPs were from coding regions, there were however several SNPs from intron regions (Table 6 and Additional file 5) suggesting that some of these SNPs may be from unspliced pre-mRNA. The intronic SNPs may also represent incomplete 
Table 4 Gene ontology based classification of differentially expressed genes GO CATEGORY

up-regulated

GO:0042221_response_to_chemical_stimulus

GO:0050896_response_to_stimulus

GO:0009266_response_to_temperature_stimulus

GO:0009408_response_to_heat

GO:0009628_response_to_abiotic_stimulus

GO:0006950_response_to_stress

GO:0009644_response_to_high_light_intensity

GO:0009642_response_to_light_intensity

GO:0000302_response_to_reactive_oxygen_species

GO:0006979_response_to_oxidative_stress

GO:0042542_response_to_hydrogen_peroxide

GO:0009725_response_to_hormone_stimulus

GO:0010033_response_to_organic_substance

GO:0009415_response_to_water

GO:0009719_response_to_endogenous_stimulus

GO:0009737_response_to_abscisic_acid_stimulus

GO:0009414_response_to_water_deprivation

GO:0010035_response_to_inorganic_substance

GO:0006970_response_to_osmotic_stress

GO:0009651_response_to_salt_stress

\section{Down-regulated}

GO:0009698_phenylpropanoid_metabolic_process

GO:0009699_phenylpropanoid_biosynthetic_process

GO:0019748_secondary_metabolic_process

GO:0007167_enzyme_linked_receptor_protein_signaling

GO:0007169_transmembrane_receptor_protein_tyrosine_kinase_signaling_pathway

GO:0007166_cell_surface_receptor_linked_signaling

GO:0009813_flavonoid_biosynthetic_process

GO:0019438_aromatic_compound_biosynthetic_process

GO:0006725_cellular_aromatic_compound_metabolic_proces

GO:0009812_flavonoid_metabolic_process

GO:0006629_lipid_metabolic_process

GO:0071555_cell_wall_organization

GO:0009653_anatomical_structure_morphogenesis

GO:0071554_cell_wall_organization_or_biogenesis

GO:0009664_plant-type_cell_wall_organization 
Table 4 Gene ontology based classification of differentially expressed genes (Continued)

\begin{tabular}{lcc}
\hline GO:0009827_plant-type_cell_wall_modification & 19 & 10 \\
GO:0070882_cellular_cell_wall_organization_or_biogenesis & 89 & 23 \\
GO:0048869_cellular_developmental_process & 323 & 54 \\
GO:0009808_lignin_metabolic_process & 35 & 13 \\
\hline
\end{tabular}

Gene ontology categories of up-regulated and down-regulated genes under water stress conditions (S1) among the most significantly differentiated transcripts. Total genes is the number of genes in a category, changed genes are the number of gene showed differential expression and FDR ( $<0.0001)$ is the one-sided Fisher's exact $p$ value corrected for multiple comparisons.

annotations of E. grandis. Ten of the intronic SNPs were within the splice sites.

\section{GO analysis of genes showing differential allelic expression}

We used GO enrichment analysis to identify the functional categories enriched among the genes that showed significant differential allelic expression. GO enrichment tests were performed separately for genes that showed significant differential allelic expression as well as total gene expression between control $(\mathrm{C} 1)$ and stress (S1) treatments and genes that showed only significant differential allelic expression but similar total gene expression between control and stress treatments. Genes that showed both allelic and total gene expression were enriched in stress and metabolic process gene categories (Additional file 6) as identified previously (Table 4). Interestingly, several stress-related gene categories (e.g. response to abiotic, salt and osmotic stress) were also enriched among the genes that showed differential allelic expression but no change in total gene expression (Additional file 6).

\section{Identification of genes under selection}

To study the evolutionary selection patterns among the genes we analysed the nonsynonymous to synonymous substitution $(\mathrm{Ka} / \mathrm{Ks})$ ratios. To estimate the $\mathrm{Ka} / \mathrm{Ks}$ ratios we combined the reads from all the populations before and after the stress treatment. We identified 194855 SNPs from coding regions of 13,719 genes using 'PoPoolation'package[25]. These SNPs were annotated as nonsynonymous or synonymous using the 'PoPoolation' package. Annotations of these variants were further confirmed by visually inspecting the tracks in integrative genomics viewer IGV [26]. The proportion of nonsynonymous to synonymous mutation rates $(\mathrm{Ka} / \mathrm{Ks})$ among the genes has ranged from 0.05 to 5.9 with a mean of 0.39 among 13,719 genes (Additional file 7). Genes with $\mathrm{Ka} / \mathrm{Ks}$ ratios below 0.5 were treated as under purifying selection while gene with $\mathrm{Ka} / \mathrm{Ks}$ ratios above 1.5 were treated as under positive selection. Most of the genes (77\%, 10630 out of 13719 genes) were under negative selection with the $\mathrm{Ka} / \mathrm{Ks}$ ratios below 0.50 . In contrast the number of genes under positive selection or under diversifying selection was small. Only $2 \%$ of the genes (287 out of 13719) were under positive selection with $\mathrm{Ka} / \mathrm{Ks}$ ratios above 1.5. To identify the gene categories enriched among the genes we conducted GO enrichment tests separately for negatively and positively selected genes. While several gene categories relating to different biological processes were enriched among the negatively selected genes $(\mathrm{Ka} / \mathrm{Ks}$ ratios $<0.20$; Additional file 8$)$, gene categories enriched among the positively selected genes were primarily related to apoptosis and cell death (Table 7).

\section{Differential gene and allelic expression of positively selected genes}

Ninety genes which showed differential expressed between control $(\mathrm{C} 1)$ and stress (S1) treatments were among the positively selected genes with $\mathrm{Ka} / \mathrm{Ks}$ ratios more than 1.5 (Table 8). While several known genes and drought stress related transcription factors such as $N A C$, ERF1 and WRKY were among the positively selected and differentially expressed genes there were however several unknown genes among the positively selected genes showing differential expression. Twenty seven SNPs from 17 positively selected genes showed differential allelic expression between S0 and S1 samples (Additional file 9). Of the 27 SNPs with differential allelic expression, $78 \%$ (21 out of 27 ) of them were nonsynonymous. Of the 17 genes which showed differential allelic -expression, four genes were differentially expressed between control (C1) and stress (S1) treatments. In three SNPs from two genes (LEA, and ketoreductase) expression of one of the two alleles was completely suppressed in S0 samples while both the alleles were expressed in S1 samples.

\section{Discussion}

We observed several (> 2500) genes differentially expressed between control and water stress conditions. The large numbers of genes observed in this study compared to other studies could be due to the higher sensitivity of RNA-seq compared to microarray analysis. The high correlation in gene expression between three populations in both control and stress treatments may be due to the same factors that led to the similarity of 
Table 5 Comparison of fold changes in gene expression between different treatments

\begin{tabular}{|c|c|c|c|c|}
\hline \multirow[t]{2}{*}{ Gene_id } & \multirow{2}{*}{$\frac{\operatorname{LogFC}^{1}}{\text { C0 vs C1 }}$} & \multirow{2}{*}{$\frac{\operatorname{LogFC}}{\text { C1 vs S1 }}$} & \multirow[t]{2}{*}{ AT_accession } & \multirow{2}{*}{$\frac{\text { TAIR gene }}{\text { annotation }}$} \\
\hline & & & & \\
\hline CUFF.28412 & -31.9 & 37.5 & AT4G27450.1 & Aluminium_induced \\
\hline Eucgr.J00493 & -34.4 & 36.4 & AT2G27510.1 & ferredoxin 3 \\
\hline Eucgr.G01843 & -31.8 & 34.3 & AT5G45180.1 & Flavin-binding \\
\hline CUFF.8283 & -31.2 & 31.8 & & unknown \\
\hline Eucgr.F00644 & -3.9 & 8.6 & & unknown \\
\hline Eucgr.H04038 & -3.0 & 7.4 & AT3G62420.1 & basicregion/leucinezipper \\
\hline Eucgr.D00606 & -2.4 & 7.2 & AT1G28330.4 & dormancy-associated \\
\hline Eucgr.I02271 & 2.4 & 7.1 & AT3G12500.1 & basicchitinase \\
\hline Eucgr.F02733 & -5.4 & 7.1 & AT1G78780.2 & pathogenesis-related \\
\hline Eucgr.H00163 & -2.8 & 6.9 & AT2G38940.1 & phosphatetransporter \\
\hline Eucgr.C03715 & -4.2 & 6.7 & & unknown \\
\hline Eucgr.E03257 & -4.4 & 6.5 & AT2G33830.2 & Dormancy/auxinassociated \\
\hline Eucgr.B03987 & -2.9 & 6.4 & AT1G60470.1 & galactinolsynthase4 \\
\hline Eucgr.F01093 & 1.9 & 6.2 & AT4G27410.3 & NAC_transcriptional_regulator \\
\hline Eucgr.B01275 & -4.9 & 6.2 & AT5G18600.1 & Thioredoxin \\
\hline Eucgr.D02645 & 3.2 & 6.0 & AT3G61890.1 & homeobox12 \\
\hline Eucgr.L01022 & -4.2 & 5.8 & ATCG00020.1 & photosystem-II_reaction_center_protein \\
\hline Eucgr.C03715 & -3.1 & 5.8 & AT5G56550.1 & oxidative_stress3 \\
\hline Eucgr.J00639 & -1.9 & 5.8 & AT5G21170.2 & 5'-AMP-activated_protein_kinase \\
\hline Eucgr.A02070 & -1.8 & 5.8 & AT3G04070.1 & NAC_domain_containing_protein \\
\hline Eucgr.F00195 & 3.4 & -33.9 & AT5G42830.1 & acyl-transferase_family_protein \\
\hline Eucgr.C04029 & 2.7 & -33.9 & AT2G24800.1 & Peroxidase \\
\hline Eucgr.A02214 & 3.5 & -34.1 & AT1G28110.2 & serine_carboxypeptidase-like \\
\hline Eucgr.C01020 & 5.0 & -34.1 & & unknown \\
\hline Eucgr.D02390 & 5.6 & -34.1 & AT5G49330.1 & myb_domain_protein \\
\hline Eucgr.F02915 & 4.4 & -34.3 & & Novel \\
\hline Eucgr.F03575 & 1.4 & -34.3 & AT1G32860.1 & Glycosyl hydrolase \\
\hline Eucgr.A02965 & 3.0 & -34.4 & AT5G17050.1 & UDP-glucosyl_transferase \\
\hline Eucgr.A02209 & 4.6 & -34.4 & AT1G28110.2 & serine_carboxy_peptidase-like45 \\
\hline Eucgr.B03602 & 1.3 & -34.4 & & unknown \\
\hline Eucgr.A02209 & 3.8 & -34.8 & AT2G33530.1 & serine_carboxypeptidase-like \\
\hline Eucgr.C03801 & 4.8 & -34.9 & AT1G67290.1 & glyoxaloxidase-related \\
\hline Eucgr.A01768 & 2.2 & -35.3 & AT4G03270.1 & Cyclin \\
\hline Eucgr.I01041 & 5.5 & -35.3 & AT3G10450.2 & serine_carboxypeptidase-like7 \\
\hline Eucgr.K02440 & 1.4 & -35.5 & AT3G53190.1 & Pectinlyase-like \\
\hline Eucgr.D02610 & 3.7 & -35.6 & AT1G73880.1 & UDP-glucosyl_transferase \\
\hline CUFF.12241 & 3.4 & -35.7 & & unknown \\
\hline Eucgr.L02741 & 5.4 & -36.1 & AT5G17040.1 & UDP-Glycosyltransferase \\
\hline Eucgr.A01963 & 5.8 & -36.5 & AT2G42840.1 & protodermal_factor \\
\hline Eucgr.F02646 & 3.4 & -38.1 & AT5G09360.1 & laccase14 \\
\hline
\end{tabular}

${ }^{1} \operatorname{logFC}-\log$ fold change.

Fold changes in the significantly differentiated genes between samples collected at the beginning $(\mathrm{CO})$ and at the end of the experiment $(\mathrm{C} 1)$ are compared with the fold changes in the significantly differentiated genes between control (C1) and stress (S1) treatments. E. grandis gene names are used when the predicted genes are mapped to $E$. grandis gene coordinates. Otherwise the predicted gene names are used with a pre-fix 'CUFF'. 
Table 6 Differential allelic expression

\begin{tabular}{|c|c|c|c|c|c|c|c|c|c|}
\hline \multirow[t]{2}{*}{ Gene_id } & \multirow{2}{*}{$\frac{\text { SNP }}{\text { position }}$} & \multicolumn{3}{|c|}{ Control } & \multicolumn{3}{|c|}{ Stress } & \multirow{2}{*}{$\frac{\text { SNP }}{\text { Annotation }}$} & \multirow{2}{*}{$\frac{\text { TAIR gene }}{\text { Annotation }}$} \\
\hline & & Allele-A & Allele-B & Freq $^{1}$ & Allele-A & Allele-B & Freq & & \\
\hline Eucgr.I01495 & 25151384 & 36 & 1 & 0.97 & 15 & 589 & 0.02 & syn & basic chitinase \\
\hline Eucgr.l02395 & 34587565 & 10 & 3 & 0.77 & 40 & 85 & 0.32 & 5'UTR & heat repeat-containing \\
\hline Eucgr.l02395 & 34586749 & 8 & 16 & 0.33 & 177 & 6804 & 0.03 & non-syn & dehydrin lea \\
\hline Eucgr.F00644 & 8551578 & 5 & 44 & 0.10 & 56 & 2036 & 0.03 & non-syn & GA requiring 3 \\
\hline Eucgr.D00606 & 11184666 & 6 & 3 & 0.67 & 33 & 266 & 0.11 & syn & dormancy-associated \\
\hline Eucgr.B03987 & 63762855 & 22 & 25 & 0.47 & 111 & 430 & 0.21 & 3'UTR & glycosyl transferase \\
\hline Eucgr.B03987 & 63762861 & 20 & 29 & 0.41 & 96 & 382 & 0.20 & 3'UTR & glycosyl transferase \\
\hline Eucgr.A02790 & 38441055 & 8 & 2 & 0.80 & 121 & 579 & 0.17 & non-syn & low-temperature-induced \\
\hline Eucgr.A02790 & 38441184 & 4 & 11 & 0.27 & 31 & 738 & 0.04 & non-syn & low-temperature-induced \\
\hline Eucgr.C01388 & 22208472 & 1114 & 155 & 0.88 & 8 & 7 & 0.53 & syn & lipid transfer protein \\
\hline Eucgr.l01393 & 24399270 & 28 & 1 & 0.97 & 39 & 158 & 0.20 & non-syn & dnaj heat shock \\
\hline Eucgr.I01393 & 24399277 & 28 & 3 & 0.90 & 40 & 162 & 0.20 & non-syn & dnaj heat shock \\
\hline Eucgr.l01393 & 24399018 & 4 & 6 & 0.40 & 205 & 11 & 0.95 & non-syn & dnaj heat shock \\
\hline Eucgr.C01031 & 16478607 & 7 & 6 & 0.54 & 101 & 748 & 0.12 & syn & unknown protein \\
\hline Eucgr.H00189 & 1874619 & 7 & 1 & 0.88 & 6 & 94 & 0.06 & syn & cellulose synthase \\
\hline Eucgr.G01188 & 19918572 & 5 & 12 & 0.29 & 24 & 390 & 0.06 & syn & ethylene-dependent \\
\hline Eucgr.K01389 & 17036132 & 49 & 19 & 0.72 & 259 & 1030 & 0.20 & syn & xerico \\
\hline Eucgr.K01389 & 17035852 & 7 & 53 & 0.12 & 34 & 1275 & 0.03 & 5'UTR & xerico \\
\hline Eucgr.K01389 & 17035854 & 21 & 34 & 0.38 & 797 & 547 & 0.59 & $5^{\prime} U T R$ & xerico \\
\hline Eucgr.K01389 & 17036141 & 16 & 51 & 0.24 & 817 & 386 & 0.68 & syn & xerico \\
\hline Eucgr.E03875 & 67744315 & 114 & 123 & 0.48 & 6 & 28 & 0.18 & non-syn & methyltransferase \\
\hline Eucgr.C02590 & 49489875 & 35 & 1 & 0.97 & 87 & 324 & 0.21 & non-syn & cipk6 \\
\hline Eucgr.C02590 & 49489315 & 14 & 0 & 1.00 & 26 & 62 & 0.30 & 5'UTR & cipk6 \\
\hline Eucgr.C02590 & 49489916 & 54 & 1 & 0.98 & 153 & 271 & 0.36 & non-syn & cipk6 \\
\hline Eucgr.C02590 & 49489924 & 55 & 2 & 0.96 & 154 & 242 & 0.39 & syn & cipk6 \\
\hline Eucgr.C02590 & 49489939 & 15 & 7 & 0.68 & 39 & 211 & 0.16 & syn & cipk6 \\
\hline Eucgr.A02077 & 31568814 & 24 & 22 & 0.52 & 177 & 435 & 0.29 & non-syn & oxidoreductase \\
\hline Eucgr.I01579 & 25696053 & 36 & 5 & 0.88 & 10 & 18 & 0.36 & syn & naringenin-chalcone synthase \\
\hline Eucgr.l01579 & 25696038 & 30 & 4 & 0.88 & 15 & 17 & 0.47 & syn & naringenin-chalcone synthase \\
\hline
\end{tabular}

${ }^{1}$ Freq - Frequency of allele-A.

SNPs with significant differential allelic expression between samples at the beginning (SO) and at the end of stress (S1) treatment. Genes with these SNPs also showed significant differential gene expression between control (C1) and stress (S1) treatments. E. grandis genes overlapping SNP positions are shown. Gene annotations are based on Arabidopsis homologs of the Eucalyptus transcripts. More details are shown in Additional file 5.

physiological and biomass traits observed between the populations in both the treatments (Table 1).

\section{$\mathrm{GO}$ analysis reveals biologically relevant genes}

Gene ontology-based tests revealed more than 100 gene categories enriched among the top most significantly differentially expressed genes. While several drought stress genes were induced by stress treatment, several cell wall and photosynthetic genes were down regulated under stress conditions. Several growth and development genes identified by comparing the control samples taken at two intervals were down regulated under stress treatment.
Up-regulation of several metabolic process genes between the control samples and down regulation of these gens under stress treatment may reflect the reduction in growth under stress conditions suggesting that these genes play a role in normal plant growth and development. These genes may therefore be used as candidate genes for growth and biomass production.

In addition to the previously reported water stress related genes, we observed several novel and/or unknown genes showing differential expression between control and stress treatments. These form a new source of candidate genes for water stress tolerance. Functional 
Table 7 Enrichment of functional gene categories among positively selected genes

\begin{tabular}{lccc}
\hline GO CATEGORY & TOTAL GENES & CHANGED GENES & FDR \\
\hline GO:0006915_apoptosis & 32 & 7 & 0.00 \\
GO:0009404_toxin_metabolic_process & 13 & 4 & 0.01 \\
GO:0009407_toxin_catabolic_process & 13 & 7 & 0.01 \\
GO:0012501_programmed_cell_death & 68 & 7 & 0.02 \\
GO:0008219_cell_death & 84 & 7 & 0.04 \\
GO:0016265_death & 84 & 12 & 0.04 \\
GO:0006952_defense_response & 254 & & 0.08 \\
\hline
\end{tabular}

analysis of these genes may reveal novel pathways of genes responding to water stress. The new gene models predicted with reference-guided mapping which are not present in E. grandis annotations (Additional file 2) may be useful for improving the annotations of E. grandis gene models.

\section{Differential allelic expression is common}

In addition to studying gene expression patterns, RNA sequencing can also reveal differences in allelic expression. Allelic expression analysis can reveal functional regulatory variants. Within an individual both alleles are subjected to same environmental conditions and feedback control. Any bias in the expression of two alleles indicates presence of nearby cis-variants. In RNA sequencing experiments read counts at the polymorphic sites provide allelic abundance and simple statistical tests of differences in read counts at polymorphic sites allow the detection of biases in allelic expression [27]. Allelic expression imbalance (AEI) is generally measured by genotyping or sequencing SNPs in individual cDNA samples [28-30]. However, high throughput sequencing methods have recently been used for studying AEI $[31,32]$. While sequencing individual samples for AEI analysis is a powerful approach for detecting subtle differences in allelic expression, it is expensive to sequence individual samples separately. Next generation sequencing of pooled samples provides cost effective method for estimating allele frequencies at genome-wide scale [33]. Pooling and sequencing RNA samples is an efficient way to detect cis-regulatory polymorphisms at genome-wide scale [34]. Sequencing RNA samples pooled from 100 adipose and islet tissues of $F_{2}$ mice, Babak et al. . [35] found several genes showing AEI. They found a significant overlap between the genes showing AEI and cis-eQTL genes obtained from microarray analysis of the same $F_{2}$ population, indicating the robustness of this approach. While differential allelic expression from RNA sequencing of pooled samples may not indicate the presence of cis-acting variants, the correlation of allelic expression with total gene expression may indicate the presence of nearby cis-acting variants.
We used pooled RNA samples to identify SNPs in this study. Allelic expression of about $52 \%$ of significant variants correlated with differential gene expression between control (C1) and stress (S1) treatments. These variants therefore may represent cis-acting regulatory variants controlling gene expression or these variants may occur in high LD with regulatory variants in adjacent intronic, untranslated and promoter sequences. Recent genomewide association studies have demonstrated that genetic variation in regulatory regions is more important than coding regions in affecting complex traits [36]. Identification of regulatory polymorphisms is therefore crucial for understanding the control of complex traits.

Allelic expression was shown to influence gene expression and phenotype in several plant species. Drought stress was shown to induce allele specific expression in barley hybrids [37]. Allelic expression may also by caused by differential methylation of alleles. In a recent study we showed that a single nucleotide polymorphism (SNP) in a cis-regulatory element affects tree phenotypic traits (cellulose and pulp yield) through changes in allelic expression caused by differential methylation of alleles [38]. SNP markers within regulatory elements can therefore affect traits by influencing the expression of genes, and could potentially be used in breeding programs to improve complex traits such as drought tolerance, growth and wood quality traits.

Enrichment of several stress responsive gene categories among the genes showing DAE and similar total gene expression between control and stress treatments indicates that these variants may be the trans-acting variants or variants influenced by mutations to transcriptional network. Similar results were reported by Tuch et al. [32]. By comparing gene expression patterns between tumour and normal tissues they identified several genes with differential allelic expression but similar total gene expression between the two types of tissues. Gene ontology tests with allelically imbalanced genes indicated enrichment of several gene categories common to the set of differentially expressed genes between tumour and normal tissues. These results indicate that allelic expression analysis may be helpful in identifying candidate 
Table 8 Positively selected genes with differential gene expression between control (C1) and stress (S1) treatments

\begin{tabular}{|c|c|c|c|c|c|c|c|}
\hline Gene_id & Non-syn_length ${ }^{1}$ & Syn_length ${ }^{2}$ & Non-syn ${ }^{3}$ & Syn $^{4}$ & $\mathrm{Ka} / \mathrm{Ks}$ & FDR & TAIR annotation \\
\hline Eucgr.B02730 & 258 & 120 & 11 & 0 & 5.6 & $7 \mathrm{E}-16$ & no-hit \\
\hline Eucgr.E04298 & 494 & 194 & 10 & 0 & 4.3 & 0.00 & unknown \\
\hline Eucgr.J02063 & 240 & 111 & 15 & 1 & 3.7 & $8 \mathrm{E}-09$ & Histone \\
\hline Eucgr.H04702 & 312 & 126 & 8 & 0 & 3.6 & $8 \mathrm{E}-12$ & beta-ketoacyl reductase 1 \\
\hline Eucgr.B02265 & 353 & 166 & 6 & 0 & 3.3 & $2 \mathrm{E}-05$ & pleckstrin homologue 1 \\
\hline Eucgr.F04064 & 129 & 60 & 6 & 0 & 3.3 & 0.01 & histone acetyltransferase \\
\hline Eucgr.H04620 & 312 & 141 & 6 & 0 & 3.2 & $6 \mathrm{E}-13$ & unknown \\
\hline Eucgr.D01191 & 841 & 398 & 12 & 1 & 3.1 & 0.01 & Ubiquitin-conjugating enzyme \\
\hline Eucgr.D01723 & 537 & 231 & 28 & 3 & 3.1 & $3 \mathrm{E}-11$ & glycine-rich protein \\
\hline Eucgr.C02248 & 151 & 65 & 6 & 0 & 3 & 0.00 & unknown \\
\hline Eucgr.A01862 & 419 & 187 & 12 & 1 & 2.9 & 0.00 & SAM \\
\hline Eucgr.H03577 & 348 & 153 & 12 & 1 & 2.9 & 0.01 & nuclear factor $Y$ \\
\hline Eucgr.D01717 & 385 & 152 & 6 & 0 & 2.8 & 0.00 & Development and Cell Death \\
\hline Eucgr.B02882 & 357 & 159 & 5 & 0 & 2.7 & 0.00 & unknown \\
\hline Eucgr.G00701 & 103 & 47 & 5 & 0 & 2.7 & 0.01 & no-hit \\
\hline Eucgr.K01312 & 362 & 163 & 5 & 0 & 2.7 & 7E-07 & Late embryogenesis abundant \\
\hline Eucgr.J03180 & 349 & 149 & 5 & 0 & 2.6 & 0.02 & unknown \\
\hline Eucgr.B03466 & 405 & 171 & 11 & 1 & 2.5 & 0.04 & unknown \\
\hline Eucgr.F00424 & 426 & 177 & 11 & 1 & 2.5 & 0.01 & nucleoside triphosphate hydrolase \\
\hline Eucgr.l01743 & 145 & 59 & 5 & 0 & 2.5 & 0.00 & unknown \\
\hline Eucgr.G01464 & 61 & 29 & 4 & 0 & 2.4 & 0.00 & no-hit \\
\hline Eucgr.C00195 & 311 & 142 & 4 & 0 & 2.3 & 0.03 & glutamine dumper \\
\hline Eucgr.K02172 & 796 & 329 & 27 & 4 & 2.3 & $2 \mathrm{E}-05$ & proteolysis \\
\hline Eucgr.D01225 & 367 & 161 & 4 & 0 & 2.2 & 0.00 & Cupredoxin superfamily protein \\
\hline Eucgr.K01587 & 505 & 233 & 18 & 3 & 2.2 & 0.00 & Calcium-binding EF-hand family protein \\
\hline Eucgr.G02486 & 272 & 112 & 4 & 0 & 2.1 & $1 \mathrm{E}-21$ & NAC-like \\
\hline Eucgr.H04886 & 638 & 265 & 9 & 1 & 2.1 & 0.03 & A20/AN1-like zinc finger \\
\hline Eucgr.l01744 & 123 & 51 & 4 & 0 & 2.1 & $1 \mathrm{E}-06$ & no-hit \\
\hline Eucgr.L02163 & 143 & 61 & 9 & 1 & 2.1 & 0.00 & TRAF-like \\
\hline Eucgr.C01903 & 219 & 90 & 4 & 0 & 2 & 7E-07 & UDP-glucosyl transferase \\
\hline Eucgr.F01425 & 160 & 65 & 4 & 0 & 2 & $2 \mathrm{E}-10$ & glutathione S-transferase \\
\hline Eucgr.l01164 & 351 & 156 & 8 & 1 & 2 & $1 \mathrm{E}-05$ & CXE12 \\
\hline Eucgr.C02013 & 720 & 331 & 28 & 6 & 1.9 & $1 \mathrm{E}-10$ & unknown \\
\hline Eucgr.E01742 & 103 & 48 & 3 & 0 & 1.9 & 0.01 & agenet domain-containing \\
\hline Eucgr.G00935 & 80 & 31 & 4 & 0 & 1.9 & 0.01 & no-hit \\
\hline Eucgr.l02449 & 307 & 134 & 12 & 2 & 1.9 & 0.02 & no-hit \\
\hline Eucgr.J03046 & 168 & 78 & 3 & 0 & 1.9 & $2 \mathrm{E}-22$ & pleiotropic drug resistance \\
\hline Eucgr.A02694 & 128 & 59 & 3 & 0 & 1.8 & $5 \mathrm{E}-17$ & UDP-glucosyl transferase \\
\hline Eucgr.B01852 & 339 & 138 & 12 & 2 & 1.8 & $1 \mathrm{E}-34$ & soybean gene regulated by cold- 2 \\
\hline Eucgr.B02741 & 317 & 142 & 7 & 1 & 1.8 & $2 \mathrm{E}-42$ & heat shock protein 17.4 \\
\hline Eucgr.D02113 & 186 & 84 & 3 & 0 & 1.8 & $1 \mathrm{E}-11$ & ethylene responsive element binding factor \\
\hline Eucgr.E00479 & 58 & 26 & 3 & 0 & 1.8 & 0.00 & unknown \\
\hline Eucgr.F03056 & 1150 & 473 & 12 & 2 & 1.8 & 0.00 & NTH/NHS family \\
\hline Eucgr.H02910 & 254 & 106 & 12 & 2 & 1.8 & 0.01 & Calcium-binding EF-hand family \\
\hline
\end{tabular}


Table 8 Positively selected genes with differential gene expression between control (C1) and stress (S1) treatments (Continued)

\begin{tabular}{|c|c|c|c|c|c|c|c|}
\hline Eucgr.A02080 & 67 & 29 & 3 & 0 & 1.7 & 0.00 & unknown \\
\hline Eucgr.B03040 & 176 & 76 & 3 & 0 & 1.7 & $9 \mathrm{E}-06$ & unknown \\
\hline Eucgr.D00574 & 477 & 208 & 3 & 0 & 1.7 & $2 \mathrm{E}-26$ & GDSL-like Lipase/Acylhydrolase \\
\hline Eucgr.D01956 & 605 & 250 & 7 & 1 & 1.7 & $2 \mathrm{E}-18$ & cytochrome P450 \\
\hline Eucgr.F03511 & 188 & 79 & 3 & 0 & 1.7 & 0.05 & no-hit \\
\hline Eucgr.H03533 & 55 & 23 & 3 & 0 & 1.7 & 0.01 & Haloacid dehalogenase-like hydrolase \\
\hline Eucgr.l00325 & 221 & 91 & 3 & 0 & 1.7 & 0.03 & disproportionating enzyme \\
\hline Eucgr.L03100 & 466 & 194 & 3 & 0 & 1.7 & $3 \mathrm{E}-08$ & cytochrome P450 \\
\hline Eucgr.B03756 & 184 & 74 & 3 & 0 & 1.6 & $1 \mathrm{E}-12$ & beta- 6 tubulin \\
\hline Eucgr.C00342 & 193 & 80 & 14 & 3 & 1.6 & 0.00 & cytochrome P450 \\
\hline Eucgr.D00603 & 111 & 46 & 3 & 0 & 1.6 & 0.00 & Yippee family \\
\hline Eucgr.E00452 & 400 & 164 & 3 & 0 & 1.6 & 0.00 & unknown \\
\hline Eucgr.E03921 & 254 & 107 & 14 & 3 & 1.6 & $4 \mathrm{E}-25$ & Cystatin/monellin \\
\hline Eucgr.E04006 & 104 & 43 & 3 & 0 & 1.6 & 0.01 & pyridoxal phosphate-dependent \\
\hline Eucgr.F00810 & 31 & 17 & 2 & 0 & 1.6 & 0.01 & RNI-like superfamily protein \\
\hline Eucgr.F02734 & 205 & 83 & 3 & 0 & 1.6 & 0.03 & unknown \\
\hline Eucgr.G02336 & 616 & 269 & 14 & 3 & 1.6 & 0.00 & unknown \\
\hline Eucgr.G02823 & 394 & 159 & 3 & 0 & 1.6 & 0.02 & para-aminobenzoate (PABA) synthase \\
\hline Eucgr.G03355 & 724 & 311 & 18 & 4 & 1.6 & $1 \mathrm{E}-19$ & NAD(P)-binding Rossmann-fold \\
\hline Eucgr.H00155 & 459 & 183 & 15 & 3 & 1.6 & $2 \mathrm{E}-13$ & glutathione S-transferase \\
\hline Eucgr.H00996 & 111 & 45 & 3 & 0 & 1.6 & $3 \mathrm{E}-06$ & WRKY DNA-binding protein \\
\hline Eucgr.H01946 & 252 & 99 & 3 & 0 & 1.6 & $2 \mathrm{E}-08$ & unknown \\
\hline Eucgr.H04711 & 189 & 75 & 3 & 0 & 1.6 & $1 \mathrm{E}-12$ & beta-ketoacyl reductase \\
\hline Eucgr.H05152 & 281 & 115 & 3 & 0 & 1.6 & 0.00 & GA200X1 \\
\hline Eucgr.K01016 & 236 & 106 & 13 & 3 & 1.6 & $6 \mathrm{E}-12$ & unknown \\
\hline Eucgr.L03046 & 171 & 78 & 6 & 1 & 1.6 & $1 \mathrm{E}-07$ & no-hit \\
\hline Eucgr.A01283 & 476 & 190 & 10 & 2 & 1.5 & $5 E-06$ & far-red elongated hypocotyl \\
\hline Eucgr.C02545 & 651 & 261 & 10 & 2 & 1.5 & 0.02 & WRKY DNA-binding protein \\
\hline Eucgr.C03738 & 53 & 19 & 3 & 0 & 1.5 & 0.00 & no-hit \\
\hline Eucgr.E00907 & 198 & 87 & 6 & 1 & 1.5 & $7 \mathrm{E}-07$ & NB-ARC domain-containing disease resistance \\
\hline Eucgr.E02617 & 80 & 40 & 2 & 0 & 1.5 & $1 \mathrm{E}-07$ & SAM \\
\hline Eucgr.E03833 & 453 & 195 & 6 & 1 & 1.5 & $6 \mathrm{E}-06$ & C2 calcium/lipid-binding \\
\hline Eucgr.F00172 & 57 & 21 & 3 & 0 & 1.5 & $3 \mathrm{E}-05$ & cytochrome P450 \\
\hline Eucgr.F01135 & 1007 & 418 & 10 & 2 & 1.5 & $5 E-09$ & NB-ARC domain-containing disease resistance \\
\hline Eucgr.F01523 & 66 & 24 & 3 & 0 & 1.5 & $4 \mathrm{E}-07$ & no-hit \\
\hline Eucgr.F01700 & 152 & 58 & 3 & 0 & 1.5 & $7 E-13$ & sterol methyltransferase 2 \\
\hline Eucgr.F04454 & 254 & 97 & 3 & 0 & 1.5 & 0.00 & unknown \\
\hline Eucgr.G00423 & 159 & 69 & 6 & 1 & 1.5 & $4 \mathrm{E}-19$ & no-hit \\
\hline Eucgr.G01862 & 673 & 317 & 15 & 4 & 1.5 & $3 \mathrm{E}-11$ & EXORDIUM like \\
\hline Eucgr.H02734 & 30 & 15 & 2 & 0 & 1.5 & $5 E-05$ & F-box family \\
\hline Eucgr.l00883 & 829 & 362 & 36 & 10 & 1.5 & $8 \mathrm{E}-20$ & no-hit \\
\hline Eucgr.I01025 & 2 & 1 & 2 & 0 & 1.5 & 0.02 & photosystem II subunit O-2 \\
\hline Eucgr.J00894 & 163 & 62 & 3 & 0 & 1.5 & $1 \mathrm{E}-17$ & Heavy metal transport/detoxification superfamily protein \\
\hline Eucgr.J02210 & 760 & 326 & 6 & 1 & 1.5 & $2 \mathrm{E}-07$ & Tetratricopeptide repeat \\
\hline
\end{tabular}


Table 8 Positively selected genes with differential gene expression between control (C1) and stress (S1) treatments (Continued)

\begin{tabular}{lrllllll}
\hline Eucgr.K01084 & 101 & 49 & 2 & 0 & 1.5 & $4 \mathrm{E}-05$ & protein kinase C substrate \\
Eucgr.L01514 & 81 & 30 & 3 & 0 & 1.5 & 0.00 & unknown \\
\hline
\end{tabular}

${ }^{1}$ nonsynonymous length ${ }^{2}$ synonymous length ${ }^{3}$ nonsynonymous sites ${ }^{4}$ synonymous sites.

genes even when total gene expression differences between the treatments are subtle.

While sequencing pooled samples is a cost effective method, pooling different samples may however introduce different biases. To verify the allelic expression results from this study these SNPs need to be sequenced or genotyped in independent samples. Similarly, the pooling method used in this study does not allow for the detection of causal variants. Sequencing or genotyping of individual samples is required to identify the causal regulatory variants [34].

\section{Evolutionary signatures of selection among the genes}

To explore the evolutionary selection patterns among the genes and to identify the mechanisms of natural selection under water stressed conditions we studied the selection signatures using $\mathrm{Ka} / \mathrm{Ks}$ estimates. Most of the genes examined in this study are under negative or purifying selection with a mean $\mathrm{Ka} / \mathrm{Ks}$ ratio of 0.39 (Additional file 7). Similar results were reported in E. grandis [19]. The average $\mathrm{Ka} / \mathrm{Ks}$ ratio observed in that study was 0.30 . In the previous study, Novaes et al. [19] have analysed 2001 genes while in the present more than 13,000 genes were analysed. This study thus provides genomewide selection patterns among the genes expressed in the leaf tissue. Most of the protein coding genes in plants and animals are in general under purifying selection indicating that these genes may have central functions and nonsynonymous mutations affecting their function have been removed by purifying selection [39]. Gene ontology (GO) enrichment tests have revealed gene categories belonging to several biological processes were enriched among the negatively selected genes (Additional file 8). Similar results were reported in $E$. grandis where genes encoding protein translation were the most significantly enriched among negatively selected genes [19]. In the present study however apoptosis and cell death categories were significantly enriched among the positively selected genes (Table 7). Nielsen et al.. [40] have also reported that apoptosis related genes were under strong positive selection among 13,731 homologous genes between human and chimpanzee lineages.

Apoptosis involves removal of cells damaged by stresses or pathogen infections through programmed cell death. Several studies in plants have shown that disease resistance genes are under strong positive selection [41]. The role of apoptosis in defence mechanisms may be the reason for positive selection acting on genes relating apoptosis and cell death. Genes relating to stress particularly disease stress evolve rapidly to adapt to changing conditions. Maintaining different alleles will help the organisms to cope with the changing conditions [42].

The estimates of $\mathrm{Ka} / \mathrm{Ks}$ ratios in the present study are influenced by coverage of the genes. The results presented here should therefore need to be further validated. Further studies using entire genome sequences of several closely related Eucalyptus species should improve the knowledge of selection patterns among different genes. With the availability of Eucalyptus reference genome sequence and the development of improved sequence analysis tools such genome-wide comparisons are now possible.

\section{Conclusions}

We identified numerous genes that are differentially expressed between control and water stressed E. camaldulensis seedlings. In addition to the previously characterised genes we observed several novel and/or unknown genes showing differential expression. Functional analysis of these genes may provide novel insights into the genetic control of drought tolerance. We also identified several SNPs in the differentially expressed genes with allelic expression of several of these variants correlating with total gene expression. The correlation of allelic expression with total gene expression indicates that these variants may be the cis-acting regulatory variants or in LD with such variants. Analysis of the selection patterns revealed enrichment of apoptosis and cell death categories among the positively selected genes. The variants identified from differential allelic expression form a valuable resource for further studies such as association studies to identify markers for drought resistance. Through this study we show that RNA-seq can be used to reveal functional markers and evolutionary selection patterns in addition to candidate genes.

\section{Methods}

\section{Glass house experiments}

Eucalyptus camaldulensis seeds were sourced from three provenances with contrasting climates: Petford (humid tropics), Katherine (dry tropics) and Mt.Isa (semi-arid tropics). Fifteen genotypes from each provenance were 
grown in pots (approximately $8 \mathrm{l}$ ) in a glass house in April 2009. Temperatures in the glasshouse were maintained at $15^{\circ} \mathrm{C}$ minimum and $30^{\circ} \mathrm{C}$ maximum. The soil surface in the pots was covered with plastic beads to reduce evaporation. After growing the plants for five months water stress was imposed on ten genotypes by maintaining the pots at $30 \%$ of field capacity for two months. The other five genotypes were watered to field capacity (controls). Stomatal conductance, photosynthetic assimilation rates and pre-dawn water potential were measured just before imposition of water stress ( $8^{\text {th }}$ of August) and during the stress period at periodic intervals from both stressed and control plants. At the same time leaf samples were taken for RNA extraction from both stressed and control plants and immediately stored at $-80^{\circ} \mathrm{C}$. Water usage was monitored throughout the experiment by weighing the pots. Two pots containing soil but no plants were also weighed, to estimate water loss by evaporation. All plants were harvested two months after imposing the stress treatment $\left(10^{\text {th }}\right.$ of October). Harvested plants were separated into roots and shoots, oven dried at $70^{\circ} \mathrm{C}$ and biomass measurements were taken.

\section{Physiological trait measurements}

Physiological measurements were taken during the experiment, at three time points: (1) immediately prior to the imposition of water stress (8/8/2009), (2) 30 days after the imposition of water stress (11/9/2009), and (3) 52 days after the imposition of water stress (9/10/2009). Pre-dawn and mid-day water potentials and osmotic potentials were measured on fully expanded young leaves using psychrometers. Measurements of stomatal conductance were taken ten days after the imposition of stress treatment using a hand-held porometer. To determine the maximum conductance, diurnal changes in stomatal conductance were measured on three plants over three days. From this analysis it was determined that maximum conductance occurred between 11.00 AM and 1.00 PM. Leaf area of all plants was measured at final harvest.

Two-way analysis of variance (ANOVA) was used to test the effects of population, treatment and the interaction between treatment and population on all the traits measured using ANOVA functions in ' $R$ ' statistical package. Pair-wise differences between the populations for the traits were tested with Tukey's post-hoc tests.

\section{RNA isolation}

Each population of 15 seedlings was divided into two groups of ten and five seedlings before collecting RNA samples. Two leaf samples from each seedling were taken before noon just before the imposition of stress on $8^{\text {th }}$ of August. Leaf samples from ten and five seedlings of each population were bulked separately before isolating RNA. Leaf samples from 10 seedlings collected at the start of the treatment were designated as "SO" and the leaf samples from five plants taken at beginning of the treatment were designated as "CO". Similarly, two leaves from each plant were collected before noon at the end of the stress treatment on $9^{\text {th }}$ of October. Leaf samples taken from the ten seedlings under stress treatment were designated as "S1" and the leaf samples taken from the five control plants at the end of the treatment were designated as "C1". Equal amounts of leaf tissue from each population were bulked before extracting RNA. In total RNA was isolated from 12 bulks, six bulks before stress treatment (SO, C0) and six bulks at the end of stress treatment (S1, C1). RNA was isolated using Chang et al., [43] method and concentrations were measured using Qubit florometer. Purified RNA samples were sent to GeneWorks for high throughput illumina sequencing. RNA sequencing libraries were prepared using total RNA. In total, five lanes of a flow cell were used for sequencing 12 libraries. Samples were sequenced with 65 base single end reads.

\section{Read mapping}

Reference-guided transcriptome mapping was performed with the reads from high throughput sequencing. Reads were assembled using the reference genome sequence of E. grandis but without using the E. grandis gene annotations i.e., annotations were developed $a b$ initio for $E$. camaldulensis. E. grandis gene models mapping to $E$. camaldulensis predicted gene models were obtained using a BED file of the predicted gene coordinates in BEDTools package [44].

The draft genome of Eucalyptus grandis (http://web. up.ac.za/eucagen) was used for reference-guided mapping of transcriptome sequencing reads. Sequencing reads from all 12 transcriptome libraries were first pooled and mapped to the Eucalyptus genome sequence scaffolds using the Bowtie [45] and TopHat [46] software packages. Bowtie was used to index the reference genome and to map sequencing reads to the indexed genome, and TopHat identified potential exon splice junctions, and mapped sequencing reads to these junctions. TopHat was run with the default parameters except for a maximum intron length of $5000 \mathrm{bp}$. The resulting alignment (in BAM file format) was used to generate transcript annotations (in gene transfer file, or GTF, format) with the Cufflinks [47] software package. Cufflinks was run with the default parameters without supplying any annotation file. Bias detection and correction to improve the accuracy of transcript abundance was used by supplying a multi fasta file of E. grandis genome. Secondly, sequencing reads from the individual libraries were mapped against the reference genome 
sequence with TopHat to obtain alignment files (BAM) for each of the 12 libraries. The BAM file from each library was analysed with the BEDTools [44] software package, which provided counts of reads mapping to different gene products (transcripts) that were represented in the annotation file (GTF). These read counts were used in statistical tests of differential expression between control and stress treatments. Read sequence and the read counts data are deposited in NCBI's Gene Expression Omnibus and are accessible through GEO series accession number GSE39369.

\section{Analysis of differential gene expression}

Differences in gene expression between different samples were tested with edgeR [48] and DESeq [49] packages using read counts from reference-guided mapping. Read counts from three populations were used as biological replicates in differential gene expression analysis. Genes expressed at very low levels (read counts $<10$ across all six libraries) were not used in analysis of differential gene expression. The model used in edgeR for testing differential gene expression was based on a negative binomial distribution. Significance tests for differential expression were based on a modified exact test. A false discovery rate (FDR) of 0.01 was used for identifying differentially expressed genes. Similar to edgeR, DESeq also uses read counts for testing differential gene expression analysis. Variance stabilized data obtained with DESeq was used to generate the heatmaps of differentially expressed genes.

To study the biological significance of differentially expressed genes, gene ontology (GO) based enrichment tests were conducted using a web based tool GOMiner [50] (http://discover.nci.nih.gov/gominer/index.jsp). For this analysis Arabidopsis homologs of transcripts were obtained by BLAST searching the Arabidopsis protein database using blastx. BLAST search was run with the parameters of maximum high scoring segment pairs (HSP) of 100, expect value for matches of 10 and the default matrix of BLOSUM62. To identify Arabidopsis homologs for gene models predicted from referenceguided transcriptome mapping, gene sequences were extracted from the Eucalyptus reference genome sequence using gene coordinates from the gene annotation file (GTF) generated using the 'Cufflinks' package. The extracted gene sequences were BLAST searched with the Arabidopsis protein database. The identified Arabidopsis homologs were used in GO enrichment tests.

\section{Identification of SNPS}

To study allelic expression SNPs from ten seedlings before the treatment ( $\mathrm{S} 0$ ) and the same ten seedlings after treatment (S1) were analysed. The BAM files generated from TopHat analysis were used for detecting SNPs.
The BAM files were used in SAMTools [51] to produce pileup files containing SNP information. Pileup files generated from SAMtools were analysed with VarScan software [52] to count the reads mapping to each allele of a variant and to estimate the allele frequencies. The following options were used in VarScan to detect the SNPs. A minimum coverage of 8 reads mapping to variant sites, minimum base phred quality of 20 and a $P$-value of 0.05 were used for SNP calling. Reads from the three control treatment libraries (S0) and reads from the three stress treatment libraries (S1) were combined for detecting SNPs. Read counts of variant alleles from control (S0) and stress treatments (S1) were used in testing for differential allelic expression using chi-squared tests. Only consistent SNPs i.e. SNPs with the same alleles from both control and stress treatment were used in the differential allelic expression analysis. SNPs with a coverage of less than 20 reads in both the treatments were not used. Significance of the differential allelic expression was based on FDR (=0.05). The BEDTools package was used to identify gene features (from GTF file) as well as E. grandis genes overlapping SNPs.

\section{Identification of genes under selection}

To study the selection patterns of genes we have estimated the proportion of nonsynonymous to synonymous substitutions $(\mathrm{Ka} / \mathrm{Ks})$. We used the PoPoolation[25] package to identify and to annotate SNP variants i.e. to determine if an SNP is nonsynonymous or synonymous This tool uses a pileup file generated from SAMTools and a gene annotation file of coding sequences (CDS) to identify and to annotate the SNP variants. We used a BAM file generated from combining the reads from before and after the stress treatments in SAMTools to generate the pileup file containing SNP information. We extracted the coding sequences (CDS) mapping to each gene by using $E$. grandis gene annotation file (gff3). We used a minimum coverage of 20 reads, a maximum coverage of 8000 , minimum phred quality of 20 and a minimum allele count of 4 for identifying the variants. The maximum coverage was based on the observed maximum SNP coverage of 7961 reads with a minimum base quality of 20 . The identity of the variants was further confirmed by visual inspection of the tracks in integrative genomics viewer (IGV)[26]. We uploaded the BAM files, the SNP position files and E. grandis gene annotation files (gff3) into IGV and visually inspected the variants from different genes to confirm the annotations. The identified nonsynonymous and synonymous SNPs were normalised by non-synonymous and synonymous lengths calculated using the 'PoPoolation' package. The average nonsynonymous length of each codon was calculated using transversion penalty of 6 . The synonymous length was calculated as 3-nonsynonymous length. The 
$\mathrm{Ka} / \mathrm{Ks}$ ratios were estimated following Novaes et al. [19] by adding a unit to both nonsynonymous and synonymous sites. To identify the gene categories enriched among the positively and negatively selected genes we conducted the GO tests by comparing the gene categories enriched among positively and negatively selected genes separately. To identify the gene categories enriched among the positively selected genes, all the genes with $\mathrm{Ka} / \mathrm{Ks}$ ratios more than 1.5 were compared with the rest of the genes. Similarly to identify the genes enriched among the negatively selected genes, all the genes with $\mathrm{Ka} / \mathrm{Ks}$ ratios less than 0.20 were compared with the rest of the genes. GOMiner package [50] was used for GO analysis of the selected genes.

\section{Additional files}

Additional file 1: Figure S1. Comparison of biomass traits between treatments. Error bars are standard errors of mean (SEM). K-Katherine; M-Mt Isa; P-Petford.

Additional file 2: Read counts and gene identities of the significantly ( $P \leq 0.05$; bonferroni correction) differentially expressed transcripts between control (C1) and stress (S1) treatments.

Additional file 3: Pearson's correlation coefficients among the reference-guided assembly read counts from 12 libraries.

Additional file 4: Gene ontology analysis of differentially expressed transcripts between control samples collected at the beginning (CO) and at the end (C1) of the treatment.

Additional file 5: Allele frequencies of the SNPs with significant differential allelic expression between samples collected at the beginning ( $\mathrm{S} 0$ ) and at the end ( $\mathrm{S} 1$ ) of stress treatments.

Additional file 6: Enrichment of functional gene categories among genes with differential allelic expression. a). Gene categories enriched among genes showing differential allelic expression (between SO and S1samples) and differential total gene expression between control (C1) and stress (S1) treatments. b). Gene categories enriched among genes showing differential allelic expression but similar total gene expression between control (C1) and stress (S1) treatments. FDR $<0.001$.

Additional file 7: Distribution of $\mathrm{Ka} / \mathrm{Ks}$ ratios among the genes. $\mathrm{Ka} /$ Ks values are based on full length CDS gene annotations from E. grandis.

Additional file 8: Gene categories enriched among genes under purifying selection. Genes with

$\mathrm{Ka} / \mathrm{Ks}$ ratio $<0.20$ were used in $\mathrm{GO}$ analysis.

Additional file 9: Differential allelic expression among positively selected genes.

\section{Abbreviations}

SNP: Single nucleotide polymorphisms; RNA-seq: RNA sequencing; GO: Gene Ontology; DAE: Differential allelic expression; LD: Linkage disequilibrium.

\section{Competing interests}

The authors declare that they have no competing interests.

\section{Authors' contributions}

BT, NS and SS conceived the research; BT designed and conducted the experiments; BT analysed the data, BT, NS and SS drafted the manuscript. All authors read and approved the final manuscript.

\section{Acknowledgements}

We thank the Australian Tree Seed Centre (ATSC) for providing the seeds of the populations used in this study. We acknowledge the technical help provided by Guanghua Huo in conducting the glass house experiment. We thank Jason Bragg for his helpful comments on the manuscript. We acknowledge the help received from the staff at The High Resolution Plant Phenomics Centre, Canberra in measuring physiological traits. We acknowledge that the E. grandis sequence data used in this study were produced by the US Department of Energy Joint Genome Institute http://www.jgi.doe.gov/ in collaboration with the user community.

\section{Author details}

${ }^{1}$ CSIRO Plant Industry, Clunies Ross Street, Acton, ACT 2601, Australia. ${ }^{2}$ ITC India, R\&D Centre, Peenya Industrial Area, No. 3, Bangalore, Karnataka 560058, India.

Received: 19 March 2012 Accepted: 20 July 2012

Published: 1 August 2012

\section{References}

1. Zhang J, Nguyen HT, Blum A: Genetic analysis of osmotic adjustment in crop plants. J Exp Bot 1999, 50(332):291-302.

2. Bohnert HJ, Sheveleva E: Plant stress adaptations - making metabolism move. Curr Opin Plant Biol 1998, 1(3):267-274.

3. Noctor G, Foyer $\mathrm{CH}$ : ASCORBATE AND GLUTATHIONE: keeping active oxygen under control. Annual Review of Plant Physiology and Plant Molecular Biology 1998, 49(1):249-279.

4. Talamè V, Ozturk NZ, Bohnert HJ, Tuberosa R: Barley transcript profiles under dehydration shock and drought stress treatments: a comparative analysis. J Exp Bot 2007, 58(2):229-240.

5. Kawaguchi R, Girke T, Bray EA, Bailey-Serres J: Differential mRNA translation contributes to gene regulation under non-stress and dehydration stress conditions in Arabidopsis thaliana. Plant J 2004, 38(5):823-839.

6. Poroyko V, Spollen W, Hejlek L, Hernandez A, LeNoble M, Davis G, Nguyen H, Springer G, Sharp R, Bohnert H: Comparing regional transcript profiles from maize primary roots under well-watered and low water potential conditions. J Exp Bot 2007, 58(2):279-289.

7. Rabbani MA, Maruyama K, Abe H, Khan MA, Katsura K, Ito Y, Yoshiwara K Seki M, Shinozaki K, Yamaguchi-Shinozaki K: Monitoring expression profiles of rice genes under cold, drought, and high-salinity stresses and abscisic acid application using cDNA microarray and RNA gel-blot analyses. Plant Physiol 2003, 133(4):1755-1767.

8. Seki M, Narusaka M, Ishida J, Nanjo T, Fujita M, Oono Y, Kamiya A, Nakajima M, Enju A, Sakurai T, et al: Monitoring the expression profiles of 7000 Arabidopsis genes under drought, cold and high-salinity stresses using a full-length CDNA microarray. Plant J 2002, 31(3):279-292.

9. Shinozaki K, Yamaguchi-Shinozaki K: Gene networks involved in drought stress response and tolerance. J Exp Bot 2007, 58(2):221-227.

10. Agarwal $P$, Agarwal $P$, Reddy M, Sopory S: Role of DREB transcription factors in abiotic and biotic stress tolerance in plants. Plant Cell Rep 2006, 25(12):1263-1274.

11. Shinozaki K, Yamaguchi-Shinozaki K, Seki M: Regulatory network of gene expression in the drought and cold stress responses. Curr Opin Plant Biol 2003, 6(5):410-417

12. Jakoby M, Weisshaar B, Dröge-Laser W, Vicente-Carbajosa J, Tiedemann J, Kroj T, Parcy F: bZIP transcription factors in Arabidopsis. Trends Plant Sci 2002, 7(3):106-111.

13. Marè C, Mazzucotelli E, Crosatti C, Francia E, Stanca A, Cattivelli L: Hv\&lt; i\&gt;-\&lt;/i\&gt;WRKY38: a new transcription factor involved in cold- and drought-response in barley. Plant Mol Bio/2004, 55(3):399-416.

14. Olsen AN, Ernst HA, Leggio LL, Skriver K: NAC transcription factors: structurally distinct, functionally diverse. Trends Plant Sci 2005, 10(2):79-87.

15. Wang Z, Gerstein M, Snyder M: RNA-Seq: a revolutionary tool for transcriptomics. Nat Rev Genet 2009, 10(1):57-63.

16. Zenoni S, Ferrarini A, Giacomelli E, Xumerle L, Fasoli M, Malerba G, Bellin D Pezzotti M, Delledonne M: Characterization of transcriptional complexity during berry development in vitis vinifera using RNA-seq. Plant Physiol 2010, 152(4):1787-1795.

17. Mizrachi E, Hefer C, Ranik M, Joubert F, Myburg A: De novo assembled expressed gene catalog of a fast-growing Eucalyptus tree produced by Illumina mRNA-Seq. BMC Genomics 2010, 11(1):681. 
18. Filichkin SA, Priest HD, Givan SA, Shen R, Bryant DW, Fox SE, Wong W-K, Mockler TC: Genome-wide mapping of alternative splicing in Arabidopsis thaliana. Genome Res 2010, 20(1):45-58.

19. Novaes E, Drost D, Farmerie W, Pappas G, Grattapaglia D, Sederoff R, Kirst M: High-throughput gene and SNP discovery in Eucalyptus grandis, an uncharacterized genome. BMC Genomics 2008, 9(1):312.

20. Koskela J, Vinceti B, Dvorak W, Bush D, Loo J, Kjaer ED, Navarro C, Pradolina C, Bordács S: The Use and exchange of forest genetic resources for food and agriculture,Background Study Paper No. 44. Rome: Commission on Genetic Resources for Food and Agriculture, Food and Agriculture Organization of the United Nations; 2010

21. Bhumibhamon S: Problems and strategies of Eucalyptus camaldulensis plantation in Thailand. In Proceedings of the Symposium on Current Development of Fast-growing Plantation Silviculture and Management in South-East Asian Region. Edited by Horng FW, Yang JC. Taipei: Taiwan Forestry research Institute; 1992:1-8.

22. Pandey D: Tropical Forest Plantation Resources. In Forest Resources Assessment 1990. Rome: Food and Agriculture Organization of the United Nations; 1985:128.

23. Bongers F, Poorter L, Hawthorne WD: The forests of Upper Guinea: Gradients in large species composition. In Biodiversity of West African forests An ecological atlas of woody plant species. Edited by Poorter $L$, Bongers F, Kouam'e FN, Hawthorne WD. Wallingford, UK: CABI International; 2004:41-52.

24. Poorter $L$, Markesteijn L: Seedling traits determine drought tolerance of tropical tree species. Biotropica 2008, 40(3):321-331.

25. Kofler R, Orozco-terWengel P, De Maio N, Pandey RV, Nolte V, Futschik A, Kosiol C, Schlötterer C: PoPoolation: a toolbox for population genetic analysis of next generation sequencing data from pooled individuals. PLoS One 2011, 6(1):e15925

26. Robinson JT, Thorvaldsdottir H, Winckler W, Guttman M, Lander ES, Getz G, Mesirov JP: Integrative genomics viewer. Nat Biotech 2011, 29(1):24-26.

27. Pastinen T: Genome-wide allele-specific analysis: insights into regulatory variation. Nat Rev Genet 2010, 11(8):533-538.

28. Pastinen T, Ge B, Gurd S, Gaudin T, Dore C, Lemire M, Lepage P, Harmsen E, Hudson TJ: Mapping common regulatory variants to human haplotypes. Hum Mol Genet 2005, 14(24):3963-3971.

29. Pastinen T, Hudson TJ: Cis-acting regulatory variation in the human genome. Science 2004, 306(5696):647-650

30. Serre D, Gurd S, Ge B, Sladek R, Sinnett D, Harmsen E, Bibikova M, Chudin E, Barker DL, Dickinson T, et al: Differential allelic expression in the human genome: a robust approach to identify genetic and epigenetic cis-Acting mechanisms regulating gene expression. PLOS Genet 2008, 4(2):e1000006.

31. Pickrell JK, Marioni JC, Pai AA, Degner JF, Engelhardt BE, Nkadori E, Veyrieras J-B, Stephens M, Gilad Y, Pritchard JK: Understanding mechanisms underlying human gene expression variation with RNA sequencing Nature 2010, 464(7289):768-772.

32. Tuch BB, Laborde RR, Xu X, Gu J, Chung CB, Monighetti CK, Stanley SJ, Olsen KD, Kasperbauer JL, Moore EJ, et al: Tumor transcriptome sequencing reveals allelic expression imbalances associated with copy number alterations. PLoS One 2010, 5(2):e9317.

33. Futschik $A$, Schlötterer $C$ : The next generation of molecular markers from massively parallel sequencing of pooled DNA samples. Genetics 2010, 186 (1):207-218.

34. Verlaan DJ, Ge B, Grundberg E, Hoberman R, Lam KCL, Koka V, Dias J, Gurd S, Martin NW, Mallmin H, et al: Targeted screening of cis-regulatory variation in human haplotypes. Genome Res 2009, 19(1):118-127.

35. Babak T, Garrett-Engele P, Armour C, Raymond C, Keller M, Chen R, Rohl C, Johnson J, Attie A, Fraser $\mathrm{H}$, et al: Genetic validation of wholetranscriptome sequencing for mapping expression affected by cisregulatory variation. BMC Genomics 2010, 11(1):473.

36. Sadee W: Regulatory polymorphisms in key candidate genes for disease susceptibility and drug response: a mandate for valid genetic biomarkers. Expert Rev Mol Diagn 2010, 10(1):9-11.

37. Von Korff M, Radovic S, Choumane W, Stamati K, Udupa SM, Grando S, Ceccarelli S, Mackay I, Powell W, Baum M, et al: Asymmetric allele-specific expression in relation to developmental variation and drought stress in barley hybrids. Plant J 2009, 59(1):14-26.

38. Thumma BR, Matheson BA, Zhang D, Meeske C, Meder R, Downes GM, Southerton SG: Identification of a cis-acting regulatory polymorphism in a eucalypt COBRA-like gene affecting cellulose content. Genetics 2009, 183(3):1153-1164.

39. Montoya-Burgos Jl: Patterns of positive selection and neutral evolution in the protein-coding genes of Tetraodon and Takifugu. PLoS One 2011, 6(9): e24800.

40. Nielsen R, Bustamante C, Clark AG, Glanowski S, Sackton TB, Hubisz MJ, Fledel-Alon A, Tanenbaum DM, Civello D, White TJ, et al: A scan for positively selected genes in the genomes of humans and chimpanzees. PLOS Biol 2005, 3(6):e170.

41. Bakker EG, Toomajian C, Kreitman M, Bergelson J: A genome-wide survey of $\mathrm{R}$ gene polymorphisms in Arabidopsis. The Plant Cell Online 2006, 18(8):1803-1818

42. da Fonseca RR, Kosiol C, Vinař T, Siepel A, Nielsen R: Positive selection on apoptosis related genes. FEBS Lett 2010, 584(3):469-476.

43. Chang S, Puryear J, Cairney J: A simple and efficient method for isolating RNA from pine trees. Plant Mol Biol Rep 1993, 11(2):113-116.

44. Quinlan AR, Hall IM: BEDTools: a flexible suite of utilities for comparing genomic features. Bioinformatics 2010, 26(6):841-842.

45. Langmead B, Trapnell C, Pop M, Salzberg S: Ultrafast and memory-efficient alignment of short DNA sequences to the human genome. Genome Biol 2009, 10(3):R25.

46. Trapnell C, Pachter L, Salzberg SL: TopHat: discovering splice junctions with RNA-seq. Bioinformatics 2009, 25(9):1105-1111.

47. Trapnell C, Williams BA, Pertea G, Mortazavi A, Kwan G, van Baren MJ, Salzberg SL, Wold BJ, Pachter L: Transcript assembly and quantification by RNA-seq reveals unannotated transcripts and isoform switching during cell differentiation. Nat Biotech 2010, 28(5):511-515.

48. Robinson MD, McCarthy DJ, Smyth GK: edgeR: a bioconductor package for differential expression analysis of digital gene expression data. Bioinformatics 2010, 26(1):139-140.

49. Anders $S$, Huber W: Differential expression analysis for sequence count data. Genome Biol 2010, 11(10):R106. doi:110.1186/gb-2010-1111-1110-r1106.

50. Zeeberg B, Feng W, Wang G, Wang M, Fojo A, Sunshine M, Narasimhan S, Kane D, Reinhold W, Lababidi S, et al: GoMiner: a resource for biological interpretation of genomic and proteomic data. Genome Biol 2003, 4(4):R28.

51. Li H, Handsaker B, Wysoker A, Fennell T, Ruan J, Homer N, Marth G, Abecasis G, Durbin R, Subgroup GPDP: The Sequence Alignment/Map format and SAMtools. Bioinformatics 2009, 25(16):2078-2079.

52. Koboldt DC, Chen K, Wylie T, Larson DE, McLellan MD, Mardis ER, Weinstock GM, Wilson RK, Ding L: VarScan: variant detection in massively parallel sequencing of individual and pooled samples. Bioinformatics 2009, 25(17):2283-2285.

\section{doi:10.1186/1471-2164-13-364}

Cite this article as: Thumma et al:: Transcriptome sequencing of Eucalyptus camaldulensis seedlings subjected to water stress reveals functional single nucleotide polymorphisms and genes under selection. BMC Genomics 2012 13:364. 